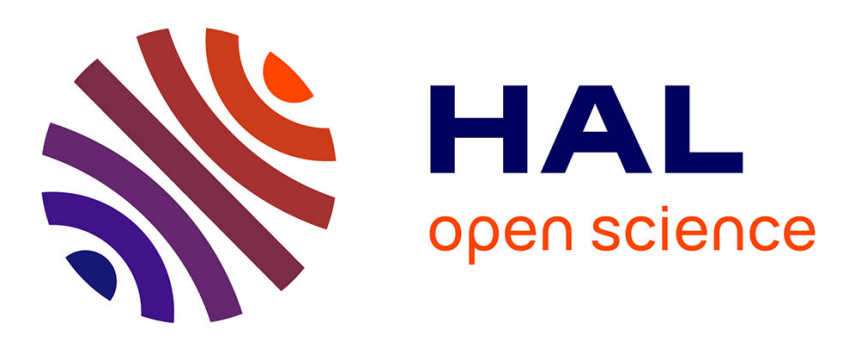

\title{
Carleman estimate for a 1D linear elastic problem involving interfaces. Application to an inverse problem.
}

\author{
Bochra Méjri
}

\section{To cite this version:}

Bochra Méjri. Carleman estimate for a 1D linear elastic problem involving interfaces. Application to an inverse problem.. 2021. hal-02398402v2

\section{HAL Id: hal-02398402 \\ https://hal.science/hal-02398402v2}

Preprint submitted on 24 Mar 2021

HAL is a multi-disciplinary open access archive for the deposit and dissemination of scientific research documents, whether they are published or not. The documents may come from teaching and research institutions in France or abroad, or from public or private research centers.
L'archive ouverte pluridisciplinaire HAL, est destinée au dépôt et à la diffusion de documents scientifiques de niveau recherche, publiés ou non, émanant des établissements d'enseignement et de recherche français ou étrangers, des laboratoires publics ou privés. 


\title{
Carleman estimate for a 1D linear elastic problem involving interfaces. Application to an inverse problem.
}

\author{
Bochra Méjri \\ Bochra.MEJRI@univ-cotedazur.fr \\ University of Côte d'Azur, CNRS, LJAD, \\ Parc Valrose, 28 Avenue Valrose, 06108 Nice Cedex 02, France
}

\begin{abstract}
The aim of this work is to study the stability of the reconstruction of some mechanical parameters that characterize interfaces within linear elastic bodies. The model considered consists of two bonded elastic solids. The adhesive elastic layer between them is a thin interphase that is approximated, by asymptotic analysis, as an interface. The resulting interface model is in turn characterized by a typical spring-type linear elastic behavior.

In this context and on a 1D configuration, we investigate a Carleman-type estimate that relates to a unique continuation principle. The proof is based on the construction of suitable weight functions: their gradients are non-zero, the jumps of the derivatives are positive across the interface and the averages of the derivatives vanish. The design of such weight functions enables the control of the interface terms in the estimates. With this at hand, we establish a Lipschitz stability estimate for the inverse problem of identifying an interface stiffness parameter from measurements that are available on both sides of the external boundary.
\end{abstract}

Keywords: interface stiffness parameter, 1D elasticity, Carleman's estimate, Lipschitz stability

\section{Introduction}

The study of solid interfaces in structural problems has garnered much scientific attention due to its meaningful role in several engineering fields (e.g. civil engineering, mechanical engineering, biomechanics, geomechanics,...). There are basically two types of solid interfaces in composite materials. A perfect interface describes the perfect (welded) contact between two solids, i.e. the displacement and the stresses are both continuous across the interface. An imperfect interface delineates the situation when there is an adhesive elastic layer of a different material (e.g. glue, lubricant,...) between the solids $[14,12,15,6,17]$.

Aim of this study is the identification of some mechanical parameters that characterize imperfect elastic interfaces. More precisely, the studied model corresponds to the sample problem of two bonded elastic solids. The adhesive elastic layer between them is a thin material interphase that is modeled as an interface by asymptotic analysis. The resulting interface model is in turn characterized by a typical springtype linear behavior. Namely, the interface tractions are assumed to be continuous, but the displacement can be discontinuous across the debonded region and, as the case may be, proportional to the interface traction via a stiffness parameter. The later is a diagonal matrix with components in the tangential and normal directions expressed in function of the thickness and the Lamé parameters of the thin elastic layer modeling the bond [15]. It is important to determine the stiffness parameters for the predications of the mechanical properties of complex systems. This parametric inverse problem is treated from two aspects: a numerical one by developing an algorithm aiming at the quantification reconstruction of the interfaces 
elastic stiffnesses, which is developed in a previous work [13] and a theoretical one by quantifying the stability of the parameters, which is presented in this paper.

Various forms of stability estimates for the Robin coefficients are developed in the literature: Both local and monotone global Lipschitz stability for regular Robin coefficient are provided by Chaabane et al. [7] under the assumption that the flux is non negative, Lipschitz stability estimate for piecewise constant Robin coefficient is proved by Sincich [18], monotone global Lipschitz stability is developed by Alessandrini et al. [1] using the three-spheres-inequalities as the main tool and also one can find global logarithmic type $[3,8]$ based on the general Carleman inequality proven by Phung [16]. We refer to the review [19] for more details about this kind of inequalities. Although, Belhachmi et al. [2] considered a Laplace equation with a Robin transmission condition, i.e. the potential is continuous and the flux is discontinuous, a dual problem for the one considered here. The proof of their directional Lipschitz stability has the same lines as the one developed in [7]. Jaoua et al. [11] proved Local Lipschitz stability for an impedance defined on a crack. Up to our knowledge, there are no studies in the literature concerning the stability issue of the interfaces elastic stiffnesses.

In this framework, we focus on the toy problem of the imperfect interface problem as a first step, avoiding the difficulties encountered with the complexity of the mathematical resolution. Therefore, we are interested in the parametric inverse problem in the case of one-dimensional elasticity equation with a spring-type linear law. More precisely, we study the stability issue of an interface elastic stiffness defined on a known point under the assumption that the data are available on both sides of the external boundaries. Roughly speaking, we seek some information on the stiffness parameters from the measurements. The reason for asking this question is that the experiment's measurements are naturally subjected to errors, so that, we are looking for a deviation of the measurements from the variations of the parameters. Thus, we concentrate on establishing a Lipschitz stability estimate, under a priori assumption on the measurements, based on Carleman's inequalities. To do so, we prove a Carleman estimate for the problem under consideration by reconstructing an appropriate weight function. Carleman estimates for parabolic equations with standard transmission conditions were proven by Doubova et al. [9] with a monotonicity assumption (i.e. the observation takes place in the region where the diffusion coefficient is the lowest) and later improved by Benabdallah et al. $[4,5]$ where the monotonicity assumption was relaxed. The proof is based on the construction of suitable weight functions, whose gradient is non-zero in the observation region and the jump of the derivative is positive across the interface. Here, we impose to these constraints that the average of the derivative of the weight function vanishes as a new parameter which enables to control the interface terms in the derivation of the Carleman-type estimate. Carleman's estimates in the $2 \mathrm{D}$ case is still an open question.

This article is outlined as follows. In the forthcoming, the forward and inverse problems of interest are reviewed and the main results are stated. Carleman inequality is established in section 3. Subsection 3.3 details the choice of the weight function by writing the quadratic form in terms of the jump and the average of the weight function gradient. The fourth section presents the stability result, based on the established Carleman inequality, for the interface stiffness parameter. The closing section is devoted to some comment.

\section{Problem setting and main results}

\subsection{The forward problem}

Consider $\Omega=(0,1) \subset \mathbb{R}$ be a bounded open set and $a$ be a point in $(0,1)$. The point $a$ split the reference domain $\Omega$ into two sub-domains $\Omega^{ \pm}$being defined as $\Omega^{-}=(0, a), \Omega^{+}=(a, 1)$. These sub-domains are characterized by the corresponding Young's moduli $E^{ \pm}$which are elements of

$$
\left\{E^{ \pm} \in \mathcal{C}^{1}\left(\Omega^{ \pm}\right) ; 0<e_{1} \leq E^{ \pm}(x) \leq e_{2}, \forall x \in \Omega^{ \pm}\right\} .
$$

Also, we denote $\Omega^{\prime}=\Omega^{+} \cup \Omega^{-}, S=\{a\}$ and $\Gamma=\{0,1\}$. 
The one-dimensional elasticity problem considered is represented by the following elliptic boundary value problem that involves a spring-type linear interface law. More precisely, the field $q$ satisfies

$$
-\partial_{x}\left(E \partial_{x} q\right)(x)=f(x), \quad x \in \Omega^{\prime} .
$$

Moreover, the interface flux is assumed to be continuous, but the field can be discontinuous across the singular point $a$ and, as the case may be, proportional to the interface flux. These conditions are represented as follows

$$
\llbracket E \partial_{x} q \rrbracket=0, \quad \llbracket q \rrbracket=k\left\langle E \partial_{x} q\right\rangle,
$$

where the jump of a quantity $q$ across a point $a$ being defined as $\llbracket q \rrbracket=q\left(a^{+}\right)-q\left(a^{-}\right)$, also the average of $q$ is defined by $\langle q\rangle=\frac{1}{2}\left(q\left(a^{+}\right)+q\left(a^{-}\right)\right)$and $k$ is a positive real-valued parameter. Besides, we impose Neumann boundary conditions on the boundary $\Gamma$.

\subsection{The inverse problem}

We are interested in the inverse problem of recovering a stiffness parameter $k$ defined on the singular point $a$ from available data on the boundary $\Gamma$ in the one-dimensional elasticity problem. Supposing $q_{i}$ as a solution satisfying $(1,2)$ associated to $k=k_{i}$, for $i=1,2$ and also $f(x)=0$ for all $x \in \Omega^{\prime}$. Here to achieve a stability result, we have to derive a Carleman-type estimate for the difference between two solutions, $q_{1}$ and $q_{2}$, (i.e. $q=q_{1}-q_{2}$ ). This difference is the solution of a non-homogeneous elliptic equation, because of the difference of the stiffness constant it does not satisfy the appropriate spring-type linear interface law, on the singular point $a$, defined above.

For this reason, we derive a peculiar Carleman-type estimate which includes additional interface terms, as follows

$$
\llbracket E \partial_{x} q \rrbracket=0, \quad \llbracket q \rrbracket=k_{2}\left\langle E \partial_{x} q\right\rangle+g,
$$

where $g=\left(k_{1}-k_{2}\right)\left\langle E \partial_{x} q_{1}\right\rangle$.

\subsection{Statement of the main results}

Here, we state our main results developed in this work. In first time, we present the following lemma specifying the suitable weight function which enables to control the interface terms in the derivation of Carleman's estimate.

Lemma 1. There exists a weight function $\beta \in C^{2}\left(\Omega^{\prime}\right)$ satisfying

$$
\llbracket \beta^{\prime} \rrbracket>0, \quad\left\langle\beta^{\prime}\right\rangle=0,
$$

for which the matrix $\mathcal{M}$ of the quadratic form is definite and positive, more precisely

$$
\operatorname{det} \mathcal{M} \sim_{s \lambda \gg} \frac{-4^{3} c_{1}^{2} c^{4} e^{2} r^{3}}{(e r-1)^{2}},
$$

where $\llbracket \beta^{\prime} \rrbracket=c(r-1),\left\langle\beta^{\prime}\right\rangle=\frac{c}{2}(r+1), c=\beta^{\prime}\left(a^{-}\right), r=\frac{\beta^{\prime}\left(a^{+}\right)}{\beta^{\prime}\left(a^{-}\right)}, c_{1}=E^{-}$and $e=\frac{E^{+}}{E^{-}}$. For example, $\beta$ has the following shape (see Figure 1)

A Carleman-type estimate for linear elastic interfaces is established. This Carleman estimate would be used later for the uniqueness and stability of the inverse solution of the parameter identification problem under consideration.

Theorem 1. There exist $\lambda_{0}>0, s_{0}>0, \varphi(x)=e^{\lambda \beta(x)}$ and a weight function $\beta$ satisfying the following assumptions

$$
\llbracket \beta^{\prime} \rrbracket>0, \quad\left\langle\beta^{\prime}\right\rangle=0,
$$




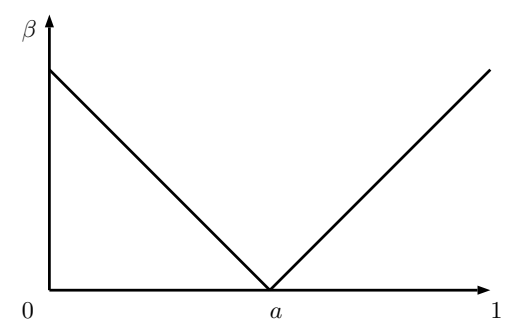

Figure 1: Typical shape's sketch for $\beta$.

so that the following estimate holds

$$
\begin{aligned}
s \lambda^{2} \int_{\Omega^{\prime}} \varphi e^{2 s \varphi}\left|\partial_{x} q\right|^{2} d x+s^{3} \lambda^{4} \int_{\Omega^{\prime}} \varphi^{3} e^{2 s \varphi}|q|^{2} d x & \leq C\left\|e^{s \varphi} f\right\|_{L^{2}\left(\Omega^{\prime}\right)}^{2}+\frac{s \lambda \varphi(a)}{k^{2}} e^{2 s \varphi(a)}|g|^{2} \\
& +s \lambda \varphi(1) e^{2 s \varphi(1)}\left|E \partial_{x} q\right|^{2}(1)+s \lambda \varphi(0) e^{2 s \varphi(0)}\left|E \partial_{x} q\right|^{2}(0) \\
& +s^{3} \lambda^{3} \varphi^{3}(1) e^{2 s \varphi(1)}|q|^{2}(1)+s^{3} \lambda^{3} \varphi^{3}(0) e^{2 s \varphi(0)}|q|^{2}(0),
\end{aligned}
$$

for all $\lambda \geq \lambda_{0}, s \geq s_{0}$, q piece-wise smooth field satisfying (1,3) and $g=\left(k_{1}-k_{2}\right)\left\langle E \partial_{x} q_{1}\right\rangle$.

The stability estimate is a fundamental result in the inverse problem which immediately yields the uniqueness result. Stability estimates are so meaningful from the theoretical aspects and also form the numerical one, more precisely this type of estimates specifies the property of the numerical algorithms. The stability estimate obtained here is based on the main global Carleman inequality which is valid only in one-dimensional.

Theorem 2. Denoting by $\Omega^{\prime}=(0, a) \cup(a, 1)$ as a union of two open subsets of $\mathbb{R}$. Moreover, one express $q_{i}$ as the solution of $(1,2)$ associated with $k=k_{i}$ and $q_{i} \in H^{1}\left(\Omega^{\prime}\right)$, for $i=1,2$. Let $m>0$ be a constant where $\left\langle E \partial_{x} q_{1}\right\rangle \geq m$ on the point $a$. Then, there exists a positive constant $\mathcal{C}$ such that

$$
\left|k_{1}-k_{2}\right|^{2} \leq \mathcal{C}\left(\left|q_{1}-q_{2}\right|^{2}(1)+\left|q_{1}-q_{2}\right|^{2}(0)\right) .
$$

This result is a very important step to numerically solve the parameters identification problem in elastic interfaces because it provides the condition in which this problem is stable numerically.

\section{Carleman inequality}

For simplicity, we divide the proof into five steps.

\subsection{Change of variables and outlines}

In this step, we set the differential equation satisfied by a new function $\psi$, which will be $q$ up to a weight function. Thus, we introduce

$$
\psi(x)=e^{s \varphi(x)} q(x), \quad \mathcal{P} \psi(x)=-e^{s \varphi(x)} \partial_{x}\left(E \partial_{x}\left(e^{-s \varphi(x)} \psi\right)\right),
$$

where $s$ is a positive constant and $\varphi$ is a positive function defined as a weight function as follows

$$
\varphi(x)=e^{\lambda \beta(x)} .
$$

Let us assume that we already find a weight function $\beta(x)$. So that, we have the following relations in $\Omega^{\prime}$ holds

$$
\partial_{x} \varphi=\lambda \beta^{\prime} \varphi
$$


We can rewrite the right-hand side of (1) by

$$
\int_{\Omega^{\prime}} f^{2} e^{2 s \varphi} \mathrm{d} x=\int_{\Omega^{\prime}}|\mathcal{P} \psi(x)|^{2} \mathrm{~d} x .
$$

Therefore, our task is a lower estimate of $\|\mathcal{P} \psi\|_{L^{2}\left(\Omega^{\prime}\right)}^{2}$. Direct calculations yield

$$
\mathcal{P} \psi=-\partial_{x}\left(E \partial_{x} \psi\right)+2 s \lambda \varphi E \beta^{\prime} \partial_{x} \psi-s^{2} \lambda^{2} \varphi^{2}\left(\beta^{\prime}\right)^{2} E \psi+s \lambda \varphi\left(E \beta^{\prime}\right)^{\prime} \psi+s \lambda^{2} \varphi E\left(\beta^{\prime}\right)^{2} \psi .
$$

For obtaining a lower estimate for $\|\mathcal{P} \psi\|_{L^{2}\left(\Omega^{\prime}\right)}^{2}$, we decompose the operator $\mathcal{P}$ into the symmetric part $\mathcal{P}_{+}$and the anti-symmetric part $\mathcal{P}_{-}$such that $\mathcal{P}=\mathcal{P}_{+}+\mathcal{P}_{-}$.

We define the adjoint operator $\mathcal{P}^{*}$ as follows

$$
\mathcal{P}^{*} \psi=-\partial_{x}\left(E \partial_{x} \psi\right)-2 s \lambda \varphi E \beta^{\prime} \partial_{x} \psi-s^{2} \lambda^{2} \varphi^{2}\left(\beta^{\prime}\right)^{2} E \psi-s \lambda \varphi\left(E \beta^{\prime}\right)^{\prime} \psi-s \lambda^{2} \varphi E\left(\beta^{\prime}\right)^{2} \psi .
$$

We define the symmetric part $\mathcal{P}_{+}$and the anti-symmetric part $\mathcal{P}_{-}$of $\mathcal{P}$ by

$$
\mathcal{P}_{+}=\frac{1}{2}\left(\mathcal{P}+\mathcal{P}^{*}\right), \quad \mathcal{P}_{-}=\frac{1}{2}\left(\mathcal{P}-\mathcal{P}^{*}\right) .
$$

Then, we have $\mathcal{P} \psi=\mathcal{P}_{+} \psi+\mathcal{P}_{-} \psi$ and

$$
\mathcal{P}_{+} \psi=-\partial_{x}\left(E \partial_{x} \psi\right)-s^{2} \lambda^{2} \varphi^{2}\left(\beta^{\prime}\right)^{2} E \psi
$$

and

$$
\mathcal{P}_{-} \psi=2 s \lambda \varphi E \beta^{\prime} \partial_{x} \psi+s \lambda \varphi\left(E \beta^{\prime}\right)^{\prime} \psi+s \lambda^{2} \varphi E\left(\beta^{\prime}\right)^{2} \psi .
$$

To get the desired estimate, we take into consideration other changes in the above decomposition as follows

$$
\begin{gathered}
\mathcal{P}_{1} \psi=\mathcal{P}_{+} \psi=-\partial_{x}\left(E \partial_{x} \psi\right)-s^{2} \lambda^{2} \varphi^{2}\left(\beta^{\prime}\right)^{2} E \psi \\
\mathcal{P}_{2} \psi=2 s \lambda \varphi E \beta^{\prime} \partial_{x} \psi+2 s \lambda^{2} \varphi E\left(\beta^{\prime}\right)^{2} \psi \\
f_{s}=e^{s \varphi} f-s \lambda \varphi\left(E \beta^{\prime}\right)^{\prime} \psi+s \lambda^{2} \varphi E\left(\beta^{\prime}\right)^{2} \psi
\end{gathered}
$$

Hence

$$
\begin{aligned}
\left\|f_{s}\right\|_{L^{2}\left(\Omega^{\prime}\right)}^{2}=\left\|\mathcal{P}_{1} \psi+\mathcal{P}_{2} \psi\right\|_{L^{2}\left(\Omega^{\prime}\right)}^{2} & =\left\|\mathcal{P}_{1} \psi\right\|_{L^{2}\left(\Omega^{\prime}\right)}^{2}+\left\|\mathcal{P}_{2} \psi\right\|_{L^{2}\left(\Omega^{\prime}\right)}^{2}+2\left(\mathcal{P}_{1} \psi, \mathcal{P}_{2} \psi\right)_{L^{2}\left(\Omega^{\prime}\right)} \\
& \geq 2\left(\mathcal{P}_{1} \psi, \mathcal{P}_{2} \psi\right)_{L^{2}\left(\Omega^{\prime}\right)} .
\end{aligned}
$$

In the following steps, we see that the definition we have made of $\varphi$ makes $\left(\mathcal{P}_{1} \psi, \mathcal{P}_{2} \psi\right)_{L^{2}\left(\Omega^{\prime}\right)}$ positive up to several terms that can be controlled whenever we make an appropriate choice of the parameters $s$ and $\lambda$.

More precisely, in the next step, we make the computations of the double products $\left(\mathcal{P}_{1} \psi, \mathcal{P}_{2} \psi\right)_{L^{2}\left(\Omega^{\prime}\right)}$. This will give an inequality with two global terms of $|\psi|^{2}$ and $\left|\partial_{x} \psi\right|^{2}$ and also an interfacial term on the left-hand side, while the observations terms will appear on the right-hand side. Finally, we will turn back to the original function $q$ and deduce the Carleman inequality.

\section{2 $\quad$ First estimates}

In this step, we estimate the right-hand side of the desired estimate from below by means of $\left(\mathcal{P}_{+} \psi, \mathcal{P}_{-} \psi\right)_{L^{2}\left(\Omega^{\prime}\right)}$. For this, we develop the four terms appearing in $\left(\mathcal{P}_{1} \psi, \mathcal{P}_{2} \psi\right)_{L^{2}\left(\Omega^{\prime}\right)}$ and we have

$$
\begin{aligned}
2\left(\mathcal{P}_{1} \psi, \mathcal{P}_{2} \psi\right)_{L^{2}\left(\Omega^{\prime}\right)} & =2\left(-\partial_{x}\left(E \partial_{x} \psi\right)-s^{2} \lambda^{2} \varphi^{2}\left(\beta^{\prime}\right)^{2} E \psi, \quad 2 s \lambda \varphi E \beta^{\prime} \partial_{x} \psi+2 s \lambda^{2} \varphi E\left(\beta^{\prime}\right)^{2} \psi\right)_{L^{2}\left(\Omega^{\prime}\right)} \\
& =2\left(-\partial_{x}\left(E \partial_{x} \psi\right), 2 s \lambda \varphi E \beta^{\prime} \partial_{x} \psi\right)_{L^{2}\left(\Omega^{\prime}\right)}+2\left(-\partial_{x}\left(E \partial_{x} \psi\right), 2 s \lambda^{2} \varphi E\left(\beta^{\prime}\right)^{2} \psi\right)_{L^{2}\left(\Omega^{\prime}\right)} \\
& +2\left(-s^{2} \lambda^{2} \varphi^{2}\left(\beta^{\prime}\right)^{2} E \psi, 2 s \lambda \varphi E \beta^{\prime} \partial_{x} \psi\right)_{L^{2}\left(\Omega^{\prime}\right)}+2\left(-s^{2} \lambda^{2} \varphi^{2}\left(\beta^{\prime}\right)^{2} E \psi, 2 s \lambda^{2} \varphi E\left(\beta^{\prime}\right)^{2} \psi\right)_{L^{2}\left(\Omega^{\prime}\right)} \\
& =2 \sum_{i, j=1}^{2} \mathcal{I}_{i j} .
\end{aligned}
$$


We reduce the orders of derivatives of $\psi$ by the integration by parts, so that we have the term $\mathcal{I}_{11}$, with the consideration $\partial_{x}\left(E \partial_{x} \psi\right) E \partial_{x} \psi=\frac{1}{2} \partial_{x}\left(\left|E \partial_{x} \psi\right|^{2}\right)$, as follows

$$
\begin{aligned}
\mathcal{I}_{11} & =-2 s \lambda \int_{\Omega^{\prime}} \varphi \beta^{\prime} \partial_{x}\left(E \partial_{x} \psi\right) E \partial_{x} \psi \mathrm{d} x \\
& =-s \lambda \int_{\Omega^{\prime}} \varphi \beta^{\prime} \partial_{x}\left(\left|E \partial_{x} \psi\right|^{2}\right) \mathrm{d} x \\
& =-s \lambda\left[\varphi \beta^{\prime}\left|E \partial_{x} \psi\right|^{2}\right]_{S \cup \Gamma}+s \lambda^{2} \int_{\Omega^{\prime}} \varphi\left(\beta^{\prime}\right)^{2}\left|E \partial_{x} \psi\right|^{2} \mathrm{~d} x+s \lambda \int_{\Omega^{\prime}} \varphi \beta^{\prime \prime}\left|E \partial_{x} \psi\right|^{2} \mathrm{~d} x,
\end{aligned}
$$

where, for a quantity $p$,

$$
[p]_{S \cup \Gamma}=p(1)-p(0)-\llbracket p \rrbracket .
$$

The term $\mathcal{I}_{11}$ is given by

$$
\mathcal{I}_{11}=-s \lambda\left[\varphi \beta^{\prime}\left|E \partial_{x} \psi\right|^{2}\right]_{S \cup \Gamma}+s \lambda^{2} \int_{\Omega^{\prime}} \varphi\left(\beta^{\prime}\right)^{2}\left|E \partial_{x} \psi\right|^{2} \mathrm{~d} x+\mathcal{X}_{11}
$$

where

$$
\mathcal{X}_{11}=s \lambda \int_{\Omega^{\prime}} \varphi \beta^{\prime \prime}\left|E \partial_{x} \psi\right|^{2} \mathrm{~d} x .
$$

Using the fact that $\partial_{x} \varphi=\lambda \beta^{\prime} \varphi$, the term $\mathcal{I}_{12}$ is given by

$$
\begin{aligned}
\mathcal{I}_{12} & =-2 s \lambda^{2} \int_{\Omega^{\prime}} \varphi E\left(\beta^{\prime}\right)^{2} \psi \partial_{x}\left(E \partial_{x} \psi\right) \mathrm{d} x \\
& =-2 s \lambda^{2}\left[\varphi E\left(\beta^{\prime}\right)^{2} \psi E \partial_{x} \psi\right]_{S \cup \Gamma}+2 s \lambda^{3} \int_{\Omega^{\prime}} \varphi E^{2}\left(\beta^{\prime}\right)^{3} \psi \partial_{x} \psi \mathrm{d} x+2 s \lambda^{2} \int_{\Omega^{\prime}} \varphi\left(E\left(\beta^{\prime}\right)^{2}\right)^{\prime} \psi E \partial_{x} \psi \mathrm{d} x \\
& +2 s \lambda^{2} \int_{\Omega^{\prime}} \varphi\left(\beta^{\prime}\right)^{2}\left|E \partial_{x} \psi\right|^{2} \mathrm{~d} x \\
& =2 s \lambda^{2} \int_{\Omega^{\prime}} \varphi\left(\beta^{\prime}\right)^{2}\left|E \partial_{x} \psi\right|^{2} \mathrm{~d} x+\mathcal{X}_{12},
\end{aligned}
$$

where

$$
\mathcal{X}_{12}=-2 s \lambda^{2}\left[\varphi E\left(\beta^{\prime}\right)^{2} \psi E \partial_{x} \psi\right]_{S \cup \Gamma}+2 s \lambda^{3} \int_{\Omega^{\prime}} \varphi E^{2}\left(\beta^{\prime}\right)^{3} \psi \partial_{x} \psi \mathrm{d} x+2 s \lambda^{2} \int_{\Omega^{\prime}} \varphi\left(E\left(\beta^{\prime}\right)^{2}\right)^{\prime} \psi E \partial_{x} \psi \mathrm{d} x .
$$

Next, noting that $2 \psi \partial_{x} \psi=\partial_{x}\left(|\psi|^{2}\right)$ and by an integration by parts, the term $\mathcal{I}_{21}$ is given by

$$
\begin{aligned}
\mathcal{I}_{21} & =-2 s^{3} \lambda^{3} \int_{\Omega^{\prime}} \varphi^{3} E^{2}\left(\beta^{\prime}\right)^{3} \psi \partial_{x} \psi \mathrm{d} x \\
& =-s^{3} \lambda^{3} \int_{\Omega^{\prime}} \varphi^{3} E^{2}\left(\beta^{\prime}\right)^{3} \partial_{x}\left(|\psi|^{2}\right) \mathrm{d} x \\
& =-s^{3} \lambda^{3}\left[\varphi^{3} E^{2}\left(\beta^{\prime}\right)^{3}|\psi|^{2}\right]_{S \cup \Gamma}+3 s^{3} \lambda^{4} \int_{\Omega^{\prime}} \varphi^{3} E^{2}\left(\beta^{\prime}\right)^{4}|\psi|^{2} \mathrm{~d} x+s^{3} \lambda^{3} \int_{\Omega^{\prime}} \varphi^{3}\left(E^{2}\left(\beta^{\prime}\right)^{3}\right)^{\prime}|\psi|^{2} \mathrm{~d} x \\
& =-s^{3} \lambda^{3}\left[\varphi^{3} E^{2}\left(\beta^{\prime}\right)^{3}|\psi|^{2}\right]_{S \cup \Gamma}+3 s^{3} \lambda^{4} \int_{\Omega^{\prime}} \varphi^{3} E^{2}\left(\beta^{\prime}\right)^{4}|\psi|^{2} \mathrm{~d} x+\mathcal{X}_{21},
\end{aligned}
$$

where

$$
\mathcal{X}_{21}=s^{3} \lambda^{3} \int_{\Omega^{\prime}} \varphi^{3}\left(E^{2}\left(\beta^{\prime}\right)^{3}\right)^{\prime}|\psi|^{2} \mathrm{~d} x
$$

The term $\mathcal{I}_{22}$ is given by

$$
\mathcal{I}_{22}=-2 s^{3} \lambda^{4} \int_{\Omega^{\prime}} \varphi^{3} E^{2}\left(\beta^{\prime}\right)^{4}|\psi|^{2} \mathrm{~d} x
$$


Therefore, noting that $s^{3}$ and $s$ are the maximum orders respectively among all the terms $\psi^{2}$ and $\left|\partial_{x} \psi\right|^{2}$ and estimating any lower order terms as non-principal terms, we have

$$
\begin{aligned}
\left(\mathcal{P}_{1} \psi, \mathcal{P}_{2} \psi\right)_{L^{2}\left(\Omega^{\prime}\right)} & =-s \lambda\left[\varphi \beta^{\prime}\left|E \partial_{x} \psi\right|^{2}\right]_{S \cup \Gamma}+s \lambda^{2} \int_{\Omega^{\prime}} \varphi\left(\beta^{\prime}\right)^{2}\left|E \partial_{x} \psi\right|^{2} \mathrm{~d} x+\mathcal{X}_{11} \\
& +2 s \lambda^{2} \int_{\Omega^{\prime}} \varphi\left(\beta^{\prime}\right)^{2}\left|E \partial_{x} \psi\right|^{2} \mathrm{~d} x+\mathcal{X}_{12} \\
& -s^{3} \lambda^{3}\left[\varphi^{3} E^{2}\left(\beta^{\prime}\right)^{3}|\psi|^{2}\right]_{S \cup \Gamma}+3 s^{3} \lambda^{4} \int_{\Omega^{\prime}} \varphi^{3} E^{2}\left(\beta^{\prime}\right)^{4}|\psi|^{2} \mathrm{~d} x+\mathcal{X}_{21} \\
& -2 s^{3} \lambda^{4} \int_{\Omega^{\prime}} \varphi^{3} E^{2}\left(\beta^{\prime}\right)^{4}|\psi|^{2} \mathrm{~d} x .
\end{aligned}
$$

Adding all the terms together to the form $\left(\mathcal{P}_{1} \psi, \mathcal{P}_{2} \psi\right)_{L^{2}\left(\Omega^{\prime}\right)}$ in $\left\|f_{s}\right\|_{L^{2}\left(\Omega^{\prime}\right)}^{2}=\left\|\mathcal{P}_{1} \psi\right\|_{L^{2}\left(\Omega^{\prime}\right)}^{2}+\left\|\mathcal{P}_{2} \psi\right\|_{L^{2}\left(\Omega^{\prime}\right)}^{2}+$ $2\left(\mathcal{P}_{1} \psi, \mathcal{P}_{2} \psi\right)_{L^{2}\left(\Omega^{\prime}\right)}$, we have

$$
\begin{aligned}
& \left\|\mathcal{P}_{1} \psi\right\|_{L^{2}\left(\Omega^{\prime}\right)}^{2}+\left\|\mathcal{P}_{2} \psi\right\|_{L^{2}\left(\Omega^{\prime}\right)}^{2}+6 s \lambda^{2} \int_{\Omega^{\prime}} \varphi\left(\beta^{\prime}\right)^{2}\left|E \partial_{x} \psi\right|^{2} \mathrm{~d} x+2 s^{3} \lambda^{4} \int_{\Omega^{\prime}} \varphi^{3} E^{2}\left(\beta^{\prime}\right)^{4}|\psi|^{2} \mathrm{~d} x \\
& -2 s \lambda\left[\varphi \beta^{\prime}\left|E \partial_{x} \psi\right|^{2}\right]_{S \cup \Gamma}-2 s^{3} \lambda^{3}\left[\varphi^{3} E^{2}\left(\beta^{\prime}\right)^{3}|\psi|^{2}\right]_{S \cup \Gamma}=\left\|f_{s}\right\|_{L^{2}\left(\Omega^{\prime}\right)}^{2}-2\left\{\mathcal{X}_{11}+\mathcal{X}_{12}+\mathcal{X}_{21}\right\} .
\end{aligned}
$$

\subsection{Choice of a weight function}

In this step, we focus our attention on the jump term at the singular point $a$ on the left-hand side of the above equality to find the appropriate weight function $\beta$ for $\mu$ as a positive definite quadratic form

$$
\mu:=s \lambda \llbracket \varphi \beta^{\prime}\left|E \partial_{x} \psi\right|^{2} \rrbracket+s^{3} \lambda^{3} \llbracket \varphi^{3} E^{2}\left(\beta^{\prime}\right)^{3}|\psi|^{2} \rrbracket .
$$

Remark 1. The perfect interface was treated in the work by Benabdallah et al [5]. They controlled the interface terms only from one side $a^{-}$due to the continuity of the interface conditions. Theirs approach fails in our case. For that reason, we propose to write the interface terms in function of the average (i.e. information from both sides). This consideration is more general and even proved by finding the same choice of the weight function for the perfect case as in the work of Benabdallah et al [5]. So, we adopt our proposed idea for the standard transmission conditions as [5] and we get the following forms

$$
\llbracket \psi \rrbracket=0, \quad \llbracket E \partial_{x} \psi \rrbracket=s \lambda \varphi \llbracket E \beta^{\prime} \rrbracket\langle\psi\rangle
$$

and

$$
\mu:=s \lambda \varphi \llbracket \beta^{\prime} \rrbracket\left\langle E \partial_{x} \psi\right\rangle^{2}+2 s^{2} \lambda^{2} \varphi^{2}\left\langle\beta^{\prime}\right\rangle \llbracket E \beta^{\prime} \rrbracket\langle\psi\rangle\left\langle E \partial_{x} \psi\right\rangle+s^{3} \lambda^{3} \varphi^{3}\left\{\llbracket E^{2}\left(\beta^{\prime}\right)^{3} \rrbracket+\frac{1}{4} \llbracket \beta^{\prime} \rrbracket \llbracket E \beta^{\prime} \rrbracket^{2}\right\}\langle\psi\rangle^{2},
$$

which gives the same choice of weight's function as [5] for the perfect interface.

Remark 2. We did the calculations for $\beta$ continuous and even discontinuous and we remark the same behaviour for both cases. To simplify the calculations, we consider $\beta$ as a continuous weight function at the point $a$.

Now, we adopt this idea for our case and for that we develop these formulas below

$$
\begin{gathered}
k\left\langle E \partial_{x} q\right\rangle=-s \lambda k \varphi(a) e^{-s \varphi(a)}\left\langle E \beta^{\prime} \psi\right\rangle+e^{-s \varphi(a)} k\left\langle E \partial_{x} \psi\right\rangle, \\
\llbracket E \partial_{x} q \rrbracket=-s \lambda \varphi(a) e^{-s \varphi(a)} \llbracket E \beta^{\prime} \psi \rrbracket+e^{-s \varphi(a)} \llbracket E \partial_{x} \psi \rrbracket .
\end{gathered}
$$

So that, we write the spring-type linear interface law verified by $\psi$ at the point $a$ using (3) under the condition $\llbracket E \beta^{\prime} \rrbracket \neq 0$ and denoting $\mathcal{Q}(a)=\left(1+\frac{1}{4} s \lambda k \varphi(a) \llbracket E \beta^{\prime} \rrbracket\right)^{-1}$

$$
\llbracket \psi \rrbracket=-s \lambda k \varphi(a) \mathcal{Q}(a)\left\langle E \beta^{\prime}\right\rangle\langle\psi\rangle+k \mathcal{Q}(a)\left\langle E \partial_{x} \psi\right\rangle+\mathcal{Q}(a) g_{s}(a)
$$


and

$\llbracket E \partial_{x} \psi \rrbracket=\left(s \lambda \varphi(a) \llbracket E \beta^{\prime} \rrbracket-s^{2} \lambda^{2} k \varphi^{2}(a) \mathcal{Q}(a)\left\langle E \beta^{\prime}\right\rangle^{2}\right)\langle\psi\rangle+s \lambda k \varphi(a) \mathcal{Q}(a)\left\langle E \beta^{\prime}\right\rangle\left\langle E \partial_{x} \psi\right\rangle+s \lambda \varphi(a) \mathcal{Q}(a)\left\langle E \beta^{\prime}\right\rangle g_{s}(a)$, where $g_{s}(a)=e^{s \varphi(a)} g(a)$ and we use the fact that

$$
\llbracket q p \rrbracket=\llbracket q \rrbracket\langle p\rangle+\langle q\rangle \llbracket p \rrbracket, \quad\langle q p\rangle=\frac{1}{4} \llbracket q \rrbracket \llbracket p \rrbracket+\langle q\rangle\langle p\rangle .
$$

After, we need the following formula in the calculation of the form $\mu$

$$
\begin{aligned}
\llbracket \psi \rrbracket^{2} & =s^{2} \lambda^{2} k^{2} \varphi^{2}(a) \mathcal{Q}^{2}(a)\left\langle E \beta^{\prime}\right\rangle^{2}\langle\psi\rangle^{2}+k^{2} \mathcal{Q}^{2}(a)\left\langle E \partial_{x} \psi\right\rangle^{2}-2 s \lambda k^{2} \varphi(a) \mathcal{Q}^{2}(a)\left\langle E \beta^{\prime}\right\rangle\langle\psi\rangle\left\langle E \partial_{x} \psi\right\rangle \\
& +\mathcal{Q}^{2}\left|g_{s}(a)\right|^{2}-2 s \lambda k \varphi(a) \mathcal{Q}^{2}(a)\left\langle E \beta^{\prime}\right\rangle\langle\psi\rangle g_{s}(a)+2 k \mathcal{Q}^{2}(a)\left\langle E \partial_{x} \psi\right\rangle g_{s}(a)
\end{aligned}
$$

and

$$
\begin{aligned}
\llbracket E \partial_{x} \psi \rrbracket^{2} & =\left(s^{2} \lambda^{2} \varphi^{2}(a) \llbracket E \beta^{\prime} \rrbracket^{2}+s^{4} \lambda^{4} k^{2} \varphi^{4}(a) \mathcal{Q}^{2}(a)\left\langle E \beta^{\prime}\right\rangle^{4}-2 s^{3} \lambda^{3} k \varphi^{3}(a) \mathcal{Q}(a) \llbracket E \beta^{\prime} \rrbracket\left\langle E \beta^{\prime}\right\rangle^{2}\right)\langle\psi\rangle^{2} \\
& +s^{2} \lambda^{2} k^{2} \varphi^{2}(a) \mathcal{Q}^{2}(a)\left\langle E \beta^{\prime}\right\rangle^{2}\left\langle E \partial_{x} \psi\right\rangle^{2} \\
& +2\left(s^{2} \lambda^{2} k \varphi^{2}(a) \mathcal{Q}(a) \llbracket E \beta^{\prime} \rrbracket\left\langle E \beta^{\prime}\right\rangle-s^{3} \lambda^{3} k^{2} \varphi^{3}(a) \mathcal{Q}^{2}(a)\left\langle E \beta^{\prime}\right\rangle^{3}\right)\langle\psi\rangle\left\langle E \partial_{x} \psi\right\rangle \\
& +s^{2} \lambda^{2} \varphi^{2}(a) \mathcal{Q}^{2}(a)\left\langle E \beta^{\prime}\right\rangle^{2}\left|g_{s}(a)\right|^{2} \\
& +2\left(s^{2} \lambda^{2} \varphi^{2}(a) \mathcal{Q}(a) \llbracket E \beta^{\prime} \rrbracket\left\langle E \beta^{\prime}\right\rangle-s^{3} \lambda^{3} k \varphi^{3}(a) \mathcal{Q}^{2}(a)\left\langle E \beta^{\prime}\right\rangle^{3}\right)\langle\psi\rangle g_{s}(a) \\
& +2 s^{2} \lambda^{2} k \varphi^{2}(a) \mathcal{Q}^{2}(a)\left\langle E \beta^{\prime}\right\rangle^{2}\left\langle E \partial_{x} \psi\right\rangle g_{s}(a) .
\end{aligned}
$$

Below, we write the form $\mu$ as a quadratic form with the fact that

$$
\llbracket p^{2} \rrbracket=2 \llbracket p \rrbracket\langle p\rangle, \quad\left\langle p^{2}\right\rangle=\frac{1}{4} \llbracket p \rrbracket^{2}+\langle p\rangle^{2} .
$$

So, the form $\mu$ is as following

$$
\begin{aligned}
\mu & =s \lambda \varphi(a) \llbracket \beta^{\prime}\left|E \partial_{x} \psi\right|^{2} \rrbracket+s^{3} \lambda^{3} \varphi^{3}(a) \llbracket E^{2}\left(\beta^{\prime}\right)^{3}|\psi|^{2} \rrbracket \\
& =s \lambda \varphi(a) \llbracket \beta^{\prime} \rrbracket\left\langle\left|E \partial_{x} \psi\right|^{2}\right\rangle+s \lambda \varphi(a)\left\langle\beta^{\prime}\right\rangle \llbracket\left|E \partial_{x} \psi\right|^{2} \rrbracket+s^{3} \lambda^{3} \varphi^{3}(a) \llbracket E^{2}\left(\beta^{\prime}\right)^{3} \rrbracket\left\langle|\psi|^{2}\right\rangle+s^{3} \lambda^{3} \varphi^{3}(a)\left\langle E^{2}\left(\beta^{\prime}\right)^{3}\right\rangle \llbracket|\psi|^{2} \rrbracket \\
& =\frac{1}{4} s \lambda \varphi(a) \llbracket \beta^{\prime} \rrbracket \llbracket E \partial_{x} \psi \rrbracket^{2}+s \lambda \varphi(a) \llbracket \beta^{\prime} \rrbracket\left\langle E \partial_{x} \psi\right\rangle^{2}+2 s \lambda \varphi(a)\left\langle\beta^{\prime}\right\rangle \llbracket E \partial_{x} \psi \rrbracket\left\langle E \partial_{x} \psi\right\rangle \\
& +\frac{1}{4} s^{3} \lambda^{3} \varphi^{3}(a) \llbracket E^{2}\left(\beta^{\prime}\right)^{3} \rrbracket \llbracket \psi \rrbracket^{2}+s^{3} \lambda^{3} \varphi^{3}(a) \llbracket E^{2}\left(\beta^{\prime}\right)^{3} \rrbracket\langle\psi\rangle^{2}+2 s^{3} \lambda^{3} \varphi^{3}(a)\left\langle E^{2}\left(\beta^{\prime}\right)^{3}\right\rangle \llbracket \psi \rrbracket\langle\psi\rangle .
\end{aligned}
$$

Using the conditions verified by $\psi$ at the singular point $a$, we get the quadratic form $\mu_{1}$ in function of the average of $\psi$ and $E \partial_{x} \psi$ as follows and the form $\mu_{2}$ in function of $g_{s}(a)$

$$
\mu=\mu_{1}+\mu_{2},
$$

where

$$
\begin{aligned}
\mu_{1} & =s \lambda \varphi(a)\left\{\frac{1}{4} s^{2} \lambda^{2} k^{2} \varphi^{2}(a) \mathcal{Q}^{2}(a) \llbracket \beta^{\prime} \rrbracket\left\langle E \beta^{\prime}\right\rangle^{2}+\llbracket \beta^{\prime} \rrbracket+2 s \lambda k \varphi(a) \mathcal{Q}(a)\left\langle\beta^{\prime}\right\rangle\left\langle E \beta^{\prime}\right\rangle\right. \\
& \left.+\frac{1}{4} s^{2} \lambda^{2} k^{2} \varphi^{2}(a) \mathcal{Q}^{2}(a) \llbracket E^{2}\left(\beta^{\prime}\right)^{3} \rrbracket\right\}\left\langle E \partial_{x} \psi\right\rangle^{2} \\
& +s^{3} \lambda^{3} \varphi^{3}(a)\left\{\frac{1}{4} \llbracket \beta^{\prime} \rrbracket \llbracket E \beta^{\prime} \rrbracket^{2}+\frac{1}{4} s^{2} \lambda^{2} k^{2} \varphi^{2}(a) \mathcal{Q}^{2}(a) \llbracket \beta^{\prime} \rrbracket\left\langle E \beta^{\prime}\right\rangle^{4}-\frac{1}{2} s \lambda k \varphi(a) \llbracket \beta^{\prime} \rrbracket \llbracket E \beta^{\prime} \rrbracket\left\langle E \beta^{\prime}\right\rangle^{2}\right\}\langle\psi\rangle^{2} \\
& +s^{3} \lambda^{3} \varphi^{3}(a)\left\{\frac{1}{4} s^{2} \lambda^{2} k^{2} \varphi^{2}(a) \mathcal{Q}^{2}(a) \llbracket E^{2}\left(\beta^{\prime}\right)^{3} \rrbracket\left\langle E \beta^{\prime}\right\rangle^{2}+\llbracket E^{2}\left(\beta^{\prime}\right)^{3} \rrbracket+-2 s \lambda k \varphi(a) \mathcal{Q}(a)\left\langle E^{2}\left(\beta^{\prime}\right)^{3}\right\rangle\left\langle E \beta^{\prime}\right\rangle\right\}\langle\psi\rangle^{2} \\
& +2 s^{2} \lambda^{2} \varphi^{2}(a)\left\{\frac{1}{4} s \lambda k \varphi(a) \mathcal{Q}(a) \llbracket \beta^{\prime} \rrbracket \llbracket E \beta^{\prime} \rrbracket\left\langle E \beta^{\prime}\right\rangle-\frac{1}{4} s^{2} \lambda^{2} k^{2} \varphi^{2}(a) \mathcal{Q}^{2}(a) \llbracket \beta^{\prime} \rrbracket\left\langle E \beta^{\prime}\right\rangle^{3}\right\}\langle\psi\rangle\left\langle E \partial_{x} \psi\right\rangle \\
& +2 s^{2} \lambda^{2} \varphi^{2}(a)\left\{\left\langle\beta^{\prime}\right\rangle \llbracket E \beta^{\prime} \rrbracket-s \lambda k \varphi(a) \mathcal{Q}(a)\left\langle\beta^{\prime}\right\rangle\left\langle E \beta^{\prime}\right\rangle^{2}-\frac{1}{4} s^{2} \lambda^{2} k^{2} \varphi^{2}(a) \mathcal{Q}^{2}(a) \llbracket E^{2}\left(\beta^{\prime}\right)^{3} \rrbracket\left\langle E \beta^{\prime}\right\rangle\right. \\
& \left.+s \lambda k \varphi(a) \mathcal{Q}(a)\left\langle E^{2}\left(\beta^{\prime}\right)^{3}\right\rangle\right\}\langle\psi\rangle\left\langle E \partial_{x} \psi\right\rangle
\end{aligned}
$$


and

$$
\begin{aligned}
\mu_{2} & =\frac{1}{4} s^{3} \lambda^{3} \varphi^{3}(a) \mathcal{Q}^{2}(a)\left(\llbracket \beta^{\prime} \rrbracket\left\langle E \beta^{\prime}\right\rangle^{2}+\llbracket E^{2}\left(\beta^{\prime}\right)^{3} \rrbracket\right)\left|g_{s}(a)\right|^{2}+2 s^{2} \lambda^{2} \varphi^{2}(a) \mathcal{Q}(a)\left\langle\beta^{\prime}\right\rangle\left\langle E \beta^{\prime}\right\rangle\left\langle E \partial_{x} \psi\right\rangle g_{s}(a) \\
& +\frac{1}{2} s^{3} \lambda^{3} k \varphi^{3}(a) \mathcal{Q}^{2}(a)\left(\llbracket \beta^{\prime} \rrbracket\left\langle E \beta^{\prime}\right\rangle^{2}+\llbracket E^{2}\left(\beta^{\prime}\right)^{3} \rrbracket\right)\left\langle E \partial_{x} \psi\right\rangle g_{s}(a) \\
& +s^{3} \lambda^{3} \varphi^{3}(a) \mathcal{Q}(a)\left(\frac{1}{2} \llbracket \beta^{\prime} \rrbracket \llbracket E \beta^{\prime} \rrbracket\left\langle E \beta^{\prime}\right\rangle+2\left\langle E^{2}\left(\beta^{\prime}\right)^{3}\right\rangle\right)\langle\psi\rangle g_{s}(a) \\
& -\frac{1}{2} s^{4} \lambda^{4} k \varphi^{4}(a) \mathcal{Q}^{2}(a)\left(\llbracket \beta^{\prime} \rrbracket\left\langle E \beta^{\prime}\right\rangle^{3}+\llbracket E^{2}\left(\beta^{\prime}\right)^{3} \rrbracket\left\langle E \beta^{\prime}\right\rangle\right)\langle\psi\rangle g_{s}(a) .
\end{aligned}
$$

We note that we can write the quadratic form $\mu_{1}$ in a matrix form as follows

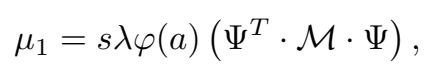

where $\Psi$ is the following vector

$$
\Psi=\left(\left\langle E \partial_{x} \psi\right\rangle, s \lambda \varphi(a)\langle\psi\rangle\right)^{T}
$$

and the coefficient of the symmetric matrix $\mathcal{M}$ are given by

$$
\begin{aligned}
\mathcal{M}_{11}= & \frac{1}{4} s^{2} \lambda^{2} k^{2} \varphi^{2}(a) \mathcal{Q}^{2}(a) \llbracket \beta^{\prime} \rrbracket\left\langle E \beta^{\prime}\right\rangle^{2}+\llbracket \beta^{\prime} \rrbracket+2 s \lambda k \varphi(a) \mathcal{Q}(a)\left\langle\beta^{\prime}\right\rangle\left\langle E \beta^{\prime}\right\rangle+\frac{1}{4} s^{2} \lambda^{2} k^{2} \varphi^{2}(a) \mathcal{Q}^{2}(a) \llbracket E^{2}\left(\beta^{\prime}\right)^{3} \rrbracket \\
\mathcal{M}_{22}= & \frac{1}{4} \llbracket \beta^{\prime} \rrbracket \llbracket E \beta^{\prime} \rrbracket^{2}+\frac{1}{4} s^{2} \lambda^{2} k^{2} \varphi^{2}(a) \mathcal{Q}^{2}(a) \llbracket \beta^{\prime} \rrbracket\left\langle E \beta^{\prime}\right\rangle^{4}-\frac{1}{2} s \lambda k \varphi(a) \mathcal{Q}(a) \llbracket \beta^{\prime} \rrbracket \llbracket E \beta^{\prime} \rrbracket\left\langle E \beta^{\prime}\right\rangle^{2} \\
& +\frac{1}{4} s^{2} \lambda^{2} k^{2} \varphi^{2}(a) \mathcal{Q}^{2}(a) \llbracket E^{2}\left(\beta^{\prime}\right)^{3} \rrbracket\left\langle E \beta^{\prime}\right\rangle^{2}+\llbracket E^{2}\left(\beta^{\prime}\right)^{3} \rrbracket-2 s \lambda k \varphi(a) \mathcal{Q}(a)\left\langle E^{2}\left(\beta^{\prime}\right)^{3}\right\rangle\left\langle E \beta^{\prime}\right\rangle \\
\mathcal{M}_{12}=\mathcal{M}_{21} & =\frac{1}{4} s \lambda k \varphi(a) \mathcal{Q}(a) \llbracket \beta^{\prime} \rrbracket \llbracket E \beta^{\prime} \rrbracket\left\langle E \beta^{\prime}\right\rangle-\frac{1}{4} s^{2} \lambda^{2} k^{2} \varphi^{2}(a) \mathcal{Q}^{2}(a) \llbracket \beta^{\prime} \rrbracket\left\langle E \beta^{\prime}\right\rangle^{3}+\left\langle\beta^{\prime}\right\rangle \llbracket E \beta^{\prime} \rrbracket \\
& -s \lambda k \varphi(a) \mathcal{Q}(a)\left\langle\beta^{\prime}\right\rangle\left\langle E \beta^{\prime}\right\rangle^{2}-\frac{1}{4} s^{2} \lambda^{2} k^{2} \varphi^{2}(a) \mathcal{Q}^{2}(a) \llbracket E^{2}\left(\beta^{\prime}\right)^{3} \rrbracket\left\langle E \beta^{\prime}\right\rangle+s \lambda k \varphi(a) \mathcal{Q}(a)\left\langle E^{2}\left(\beta^{\prime}\right)^{3}\right\rangle .
\end{aligned}
$$

The determinant of the matrix $\mathcal{M}$ is calculated by considering $\mathcal{A}=s \lambda k \varphi(a) \mathcal{Q}(a)$

$$
\begin{aligned}
\operatorname{det} \mathcal{M} & =\mathcal{M}_{11} \mathcal{M}_{22}-\mathcal{M}_{12}^{2} \\
& =\frac{1}{2} \mathcal{A}^{2} \llbracket \beta^{\prime} \rrbracket\left\langle E \beta^{\prime}\right\rangle^{2} \llbracket E^{2}\left(\beta^{\prime}\right)^{3} \rrbracket+\frac{1}{4} \llbracket \beta^{\prime} \rrbracket^{2} \llbracket E \beta^{\prime} \rrbracket^{2}+\frac{1}{4} \mathcal{A}^{2} \llbracket \beta^{\prime} \rrbracket^{2}\left\langle E \beta^{\prime}\right\rangle^{4}-\frac{1}{2} \mathcal{A} \llbracket \beta^{\prime} \rrbracket^{2} \llbracket E \beta^{\prime} \rrbracket\left\langle E \beta^{\prime}\right\rangle^{2}+\llbracket \beta^{\prime} \rrbracket \llbracket E^{2}\left(\beta^{\prime}\right)^{3} \rrbracket \\
& -2 \mathcal{A} \llbracket \beta^{\prime} \rrbracket\left\langle E \beta^{\prime}\right\rangle\left\langle E^{2}\left(\beta^{\prime}\right)^{3}\right\rangle+2 \mathcal{A} \llbracket E^{2}\left(\beta^{\prime}\right)^{3} \rrbracket\left\langle\beta^{\prime}\right\rangle\left\langle E \beta^{\prime}\right\rangle-2 \mathcal{A}^{2}\left\langle\beta^{\prime}\right\rangle\left\langle E \beta^{\prime}\right\rangle^{2}\left\langle E^{2}\left(\beta^{\prime}\right)^{3}\right\rangle+\frac{1}{16} \mathcal{A}^{2} \llbracket \beta^{\prime} \rrbracket \llbracket E \beta^{\prime} \rrbracket^{2} \llbracket E^{2}\left(\beta^{\prime}\right)^{3} \rrbracket \\
& +\frac{1}{4} \mathcal{A}^{2} \llbracket E^{2}\left(\beta^{\prime}\right)^{3} \rrbracket^{2}-\llbracket E \beta^{\prime} \rrbracket^{2}\left\langle\beta^{\prime}\right\rangle^{2}-\mathcal{A}^{2}\left\langle\beta^{\prime}\right\rangle^{2}\left\langle E \beta^{\prime}\right\rangle^{4}-\mathcal{A}^{2}\left\langle E^{2}\left(\beta^{\prime}\right)^{3}\right\rangle^{2}-\frac{1}{2} \mathcal{A}^{2} \llbracket \beta^{\prime} \rrbracket \llbracket E \beta^{\prime} \rrbracket\left\langle E \beta^{\prime}\right\rangle\left\langle E^{2}\left(\beta^{\prime}\right)^{3}\right\rangle \\
& +2 \mathcal{A} \llbracket E \beta^{\prime} \rrbracket\left\langle\beta^{\prime}\right\rangle^{2}\left\langle E \beta^{\prime}\right\rangle^{2}+\frac{1}{2} \mathcal{A}^{2} \llbracket E \beta^{\prime} \rrbracket \llbracket E^{2}\left(\beta^{\prime}\right)^{3} \rrbracket\left\langle\beta^{\prime}\right\rangle\left\langle E \beta^{\prime}\right\rangle-2 \mathcal{A} \llbracket E \beta^{\prime} \rrbracket\left\langle\beta^{\prime}\right\rangle\left\langle E^{2}\left(\beta^{\prime}\right)^{3}\right\rangle .
\end{aligned}
$$

Developing all the terms of $\llbracket E^{2}\left(\beta^{\prime}\right)^{3} \rrbracket$ and $\left\langle E^{2}\left(\beta^{\prime}\right)^{3}\right\rangle$ as follows

$$
\begin{aligned}
& \llbracket E^{2}\left(\beta^{\prime}\right)^{3} \rrbracket=\frac{1}{4} \llbracket \beta^{\prime} \rrbracket \llbracket E \beta^{\prime} \rrbracket^{2}+\llbracket \beta^{\prime} \rrbracket\left\langle E \beta^{\prime}\right\rangle^{2}+2 \llbracket E \beta^{\prime} \rrbracket\left\langle\beta^{\prime}\right\rangle\left\langle E \beta^{\prime}\right\rangle, \\
& \left\langle E^{2}\left(\beta^{\prime}\right)^{3}\right\rangle=\frac{1}{2} \llbracket \beta^{\prime} \rrbracket \llbracket E \beta^{\prime} \rrbracket\left\langle E \beta^{\prime}\right\rangle+\frac{1}{4} \llbracket E \beta^{\prime} \rrbracket^{2}\left\langle\beta^{\prime}\right\rangle+\left\langle\beta^{\prime}\right\rangle\left\langle E \beta^{\prime}\right\rangle^{2},
\end{aligned}
$$

the determinant of the matrix $\mathcal{M}$ is then given by

$$
\begin{aligned}
\operatorname{det} \mathcal{M}= & -\frac{3}{16} \mathcal{A}^{2} \llbracket \beta^{\prime} \rrbracket^{2} \llbracket E \beta^{\prime} \rrbracket^{2}\left\langle E \beta^{\prime}\right\rangle^{2}+\mathcal{A}^{2} \llbracket \beta^{\prime} \rrbracket^{2}\left\langle E \beta^{\prime}\right\rangle^{4}+\frac{1}{2} \llbracket \beta^{\prime} \rrbracket^{2} \llbracket E \beta^{\prime} \rrbracket^{2}-\frac{3}{2} \mathcal{A} \llbracket \beta^{\prime} \rrbracket^{2} \llbracket E \beta^{\prime} \rrbracket\left\langle E \beta^{\prime}\right\rangle^{2}+\llbracket \beta^{\prime} \rrbracket^{2}\left\langle E \beta^{\prime}\right\rangle^{2} \\
& +2 \llbracket \beta^{\prime} \rrbracket \llbracket E \beta^{\prime} \rrbracket\left\langle\beta^{\prime}\right\rangle\left\langle E \beta^{\prime}\right\rangle-\mathcal{A} \llbracket \beta^{\prime} \rrbracket \llbracket E \beta^{\prime} \rrbracket^{2}\left\langle\beta^{\prime}\right\rangle\left\langle E \beta^{\prime}\right\rangle+4 \mathcal{A} \llbracket E \beta^{\prime} \rrbracket\left\langle\beta^{\prime}\right\rangle^{2}\left\langle E \beta^{\prime}\right\rangle^{2}+\mathcal{A}^{2} \llbracket E \beta^{\prime} \rrbracket^{2}\left\langle\beta^{\prime}\right\rangle^{2}\left\langle E \beta^{\prime}\right\rangle^{2} \\
& -4 \mathcal{A}^{2}\left\langle\beta^{\prime}\right\rangle^{2}\left\langle E \beta^{\prime}\right\rangle^{4}-\frac{1}{16} \mathcal{A}^{2} \llbracket E \beta^{\prime} \rrbracket^{4}\left\langle\beta^{\prime}\right\rangle^{2}+\frac{1}{32} \mathcal{A}^{2} \llbracket \beta^{\prime} \rrbracket^{2} \llbracket E \beta^{\prime} \rrbracket^{4}+\frac{1}{8} \mathcal{A}^{2} \llbracket \beta^{\prime} \rrbracket \llbracket E \beta^{\prime} \rrbracket^{3}\left\langle\beta^{\prime}\right\rangle\left\langle E \beta^{\prime}\right\rangle-\llbracket E \beta^{\prime} \rrbracket^{2}\left\langle\beta^{\prime}\right\rangle^{2} \\
& -\frac{1}{2} \mathcal{A} \llbracket E \beta^{\prime} \rrbracket^{3}\left\langle\beta^{\prime}\right\rangle^{2} .
\end{aligned}
$$


The determinant $\operatorname{det} \mathcal{M}$ is given as follows

$\operatorname{det} \mathcal{M} \sim_{s \lambda \gg} \llbracket \beta^{\prime} \rrbracket^{2} \llbracket E \beta^{\prime} \rrbracket^{2}-4 \llbracket E \beta^{\prime} \rrbracket^{2}\left\langle\beta^{\prime}\right\rangle^{2}-8 \llbracket \beta^{\prime} \rrbracket^{2}\left\langle E \beta^{\prime}\right\rangle^{2}+32\left\langle\beta^{\prime}\right\rangle^{2}\left\langle E \beta^{\prime}\right\rangle^{2}+16 \frac{\llbracket \beta^{\prime} \rrbracket^{2}\left\langle E \beta^{\prime}\right\rangle^{4}}{\llbracket E \beta^{\prime} \rrbracket^{2}}-64 \frac{\left\langle\beta^{\prime}\right\rangle^{2}\left\langle E \beta^{\prime}\right\rangle^{4}}{\llbracket E \beta^{\prime} \rrbracket^{2}}$,

with the fact that $\mathcal{A} \sim \frac{4}{\llbracket E \beta^{\prime} \rrbracket}$ for $s \lambda \gg$ (i.e. large enough).

We remark that the determinant of the matrix $\mathcal{M}$ is a polynomial of fourth order with the consideration of the following changes

$$
\llbracket \beta^{\prime} \rrbracket=c(r-1), \quad\left\langle\beta^{\prime}\right\rangle=\frac{c}{2}(r+1), \quad c=\beta^{\prime}\left(a^{-}\right), \quad r=\frac{\beta^{\prime}\left(a^{+}\right)}{\beta^{\prime}\left(a^{-}\right)}, \quad c_{1}=E^{-}, \quad e=\frac{E^{+}}{E^{-}} .
$$

Finally, the determinant is as follows

$$
\begin{aligned}
\operatorname{det} \mathcal{M} & \sim_{s \lambda \gg} c_{1}^{2} c^{4}\left(-4 r(e r-1)^{2}+8 r(e r+1)^{2}-4 r \frac{(e r+1)^{4}}{(e r-1)^{2}}\right) \\
& =\frac{-4^{3} c_{1}^{2} c^{4} e^{2} r^{3}}{(e r-1)^{2}}
\end{aligned}
$$

The determinant of the matrix $\mathcal{M}$ is given in function of its variable $r$. Here, one needs to seek the properties of the weight function (i.e. the values of the variable $r$ ) for which the determinant is positive. In this case, we can choose $r=-1$ which gives $\beta^{\prime}\left(a^{+}\right)=-\beta^{\prime}\left(a^{-}\right)$. This choice explains that one needs to observe from both sides of the exterior boundary to control the interface terms. Finally, one chooses the derivative of the weight function as follows

$$
\llbracket \beta^{\prime} \rrbracket=\alpha>0, \quad\left\langle\beta^{\prime}\right\rangle=0 .
$$

It is easy to verify that $\mathcal{M}_{11}$ is a positive term with these properties of the chosen weight function. So, the form $\mu_{1}$ is a definite positive form as follows

$$
\mu_{1} \geq \mathcal{C} s \lambda \varphi(a)\left\langle E \partial_{x} \psi\right\rangle^{2}+\mathcal{C} s^{3} \lambda^{3} \varphi^{3}(a)\langle\psi\rangle^{2}
$$

\subsection{Final estimates}

The final step will be to estimate the terms on the right-hand side of the following equation. So, we obtain after the consideration of the weight function choice

$$
\begin{aligned}
& \left\|\mathcal{P}_{1} \psi\right\|_{L^{2}\left(\Omega^{\prime}\right)}^{2}+\left\|\mathcal{P}_{2} \psi\right\|_{L^{2}\left(\Omega^{\prime}\right)}^{2}+6 s \lambda^{2} \int_{\Omega^{\prime}} \varphi\left(\beta^{\prime}\right)^{2}\left|E \partial_{x} \psi\right|^{2} \mathrm{~d} x+2 s^{3} \lambda^{4} \int_{\Omega^{\prime}} \varphi^{3} E^{2}\left(\beta^{\prime}\right)^{4}|\psi|^{2} \mathrm{~d} x \\
& +\mathcal{C} s \lambda \varphi(a)\left\langle E \partial_{x} \psi\right\rangle^{2}+\mathcal{C} s^{3} \lambda^{3} \varphi^{3}(a)\langle\psi\rangle^{2}+\frac{1}{2} s^{3} \lambda^{3} \varphi^{3}(a) \mathcal{Q}^{2}(a)\left(2 \llbracket \beta^{\prime} \rrbracket\left\langle E \beta^{\prime}\right\rangle^{2}+\frac{1}{4} \llbracket \beta^{\prime} \rrbracket \llbracket E \beta^{\prime} \rrbracket^{2}\right)\left|g_{s}(a)\right|^{2} \\
& \leq\left\|f_{s}\right\|_{L^{2}\left(\Omega^{\prime}\right)}^{2}-2\left\{\mathcal{X}_{11}+\mathcal{X}_{12}+\mathcal{X}_{21}+\mathcal{Y}_{1}+\mathcal{Y}_{2}\right\}+2 s \lambda \varphi(1) \beta^{\prime}(1)\left|E \partial_{x} \psi\right|^{2}(1)-2 s \lambda \varphi(0) \beta^{\prime}(0)\left|E \partial_{x} \psi\right|^{2}(0) \\
& +2 s^{3} \lambda^{3} \varphi^{3}(1)\left(E^{2}\left(\beta^{\prime}\right)^{3}\right)(1)|\psi|^{2}(1)-2 s^{3} \lambda^{3} \varphi^{3}(0)\left(E^{2}\left(\beta^{\prime}\right)^{3}\right)(0)|\psi|^{2}(0),
\end{aligned}
$$

where

$$
\mathcal{Y}_{1}=\frac{1}{2} s^{3} \lambda^{3} k \varphi^{3}(a) \mathcal{Q}^{2}(a)\left(2 \llbracket \beta^{\prime} \rrbracket\left\langle E \beta^{\prime}\right\rangle^{2}+\frac{1}{4} \llbracket \beta^{\prime} \rrbracket \llbracket E \beta^{\prime} \rrbracket^{2}\right)\left\langle E \partial_{x} \psi\right\rangle g_{s}(a)
$$

and

$\mathcal{Y}_{2}=\frac{3}{2} s^{3} \lambda^{3} \varphi^{3}(a) \mathcal{Q}(a) \llbracket \beta^{\prime} \rrbracket \llbracket E \beta^{\prime} \rrbracket\left\langle E \beta^{\prime}\right\rangle\langle\psi\rangle g_{s}(a)-\frac{1}{2} s^{4} \lambda^{4} k \varphi^{4}(a) \mathcal{Q}^{2}(a)\left\langle E \beta^{\prime}\right\rangle\left(2 \llbracket \beta^{\prime} \rrbracket\left\langle E \beta^{\prime}\right\rangle^{2}+\frac{1}{4} \llbracket \beta^{\prime} \rrbracket \llbracket E \beta^{\prime} \rrbracket \rrbracket^{2}\right)\langle\psi\rangle g_{s}(a)$. 
Remark 3. Observe that the coefficients in front of the trace terms at 0 and 1 on the r.h.s. in the inequality (4) are positive because of the properties of the function $\beta$.

Now, we estimate the right-hand side terms in the above inequality (4) using the properties of the derivative of $\beta$ and of the Young moduli $E$ as follows

$$
\begin{gathered}
\left|\mathcal{X}_{11}\right|=\left.\left.\left|s \lambda \int_{\Omega^{\prime}} \varphi \beta^{\prime \prime}\right| E \partial_{x} \psi\right|^{2} \mathrm{~d} x\left|\leq C\left(\alpha_{1}, e_{2}\right) s \lambda \int_{\Omega^{\prime}} \varphi\right| \partial_{x} \psi\right|^{2} \mathrm{~d} x, \quad C\left(\alpha_{1}, e_{2}\right)=\alpha_{1} e_{2}^{2}, \\
\left|\mathcal{X}_{21}\right|=\left.\left.s^{3} \lambda^{3}\left|\int_{\Omega^{\prime}} \varphi^{3}\left(E^{2}\left(\beta^{\prime}\right)^{3}\right)^{\prime}\right| \psi\right|^{2} \mathrm{~d} x\left|\leq C\left(\alpha_{1}, e_{2}\right) s^{3} \lambda^{3} \int_{\Omega^{\prime}} \varphi^{3}\right| \psi\right|^{2} \mathrm{~d} x, \quad C\left(\alpha_{1}, e_{2}\right)=3 e_{2}^{2} \alpha_{1} \alpha^{2} \quad \alpha_{1}=\sup _{x \in \Omega^{\prime}}\left|\beta^{\prime \prime}\right|
\end{gathered}
$$

We remark that the second term of the following equation absorb the third one

$$
\left|\mathcal{X}_{12}\right|=\left|-2 s \lambda^{2}\left[\varphi E\left(\beta^{\prime}\right)^{2} \psi E \partial_{x} \psi\right]_{S \cup \Gamma}+2 s \lambda^{3} \int_{\Omega^{\prime}} \varphi E^{2}\left(\beta^{\prime}\right)^{3} \psi \partial_{x} \psi \mathrm{d} x+2 s \lambda^{2} \int_{\Omega^{\prime}} \varphi\left(E\left(\beta^{\prime}\right)^{2}\right)^{\prime} \psi E \partial_{x} \psi \mathrm{d} x\right|
$$

and we estimate it by the Young inequality $m n \leq \frac{m^{2}}{2 \epsilon}+\frac{\epsilon n^{2}}{2}$ as follows

$$
2 s \lambda^{3} \int_{\Omega^{\prime}} \varphi\left|E^{2}\left(\beta^{\prime}\right)^{3} \psi \partial_{x} \psi\right| \mathrm{d} x \leq C_{\epsilon}\left(\alpha, e_{2}, \epsilon\right) s \lambda^{4} \int_{\Omega^{\prime}} \varphi|\psi|^{2} \mathrm{~d} x+\epsilon s \lambda^{2} \int_{\Omega^{\prime}} \varphi\left|\partial_{x} \psi\right|^{2} \mathrm{~d} x
$$

where

$$
C_{\epsilon}\left(\alpha, e_{2}, \epsilon\right)=\frac{e_{2}^{2} \alpha^{3}}{\epsilon}
$$

We develop the first term in $\mathcal{X}_{12}$ using the formula for the jump of function's product

$$
\begin{aligned}
& -2 s \lambda^{2}\left[\varphi E\left(\beta^{\prime}\right)^{2} \psi E \partial_{x} \psi\right]_{S \cup \Gamma} \\
& =-2 s \lambda^{2} \varphi(1)\left(E\left(\beta^{\prime}\right)^{2}\right)(1) \psi(1)\left(E \partial_{x} \psi\right)(1)+2 s \lambda^{2} \varphi(0)\left(E\left(\beta^{\prime}\right)^{2}\right)(0) \psi(0)\left(E \partial_{x} \psi\right)(0)+2 s \lambda^{2} \varphi(a) \llbracket E\left(\beta^{\prime}\right)^{2} \psi E \partial_{x} \psi \rrbracket \\
& =-2 s \lambda^{2} \varphi(1)\left(E\left(\beta^{\prime}\right)^{2}\right)(1) \psi(1)\left(E \partial_{x} \psi\right)(1)+2 s \lambda^{2} \varphi(0)\left(E\left(\beta^{\prime}\right)^{2}\right)(0) \psi(0)\left(E \partial_{x} \psi\right)(0)+\frac{1}{2} s \lambda^{2} \varphi(a) \llbracket E\left(\beta^{\prime}\right)^{2} \rrbracket \llbracket \psi \rrbracket \llbracket E \partial_{x} \psi \rrbracket \\
& +2 s \lambda^{2} \varphi(a) \llbracket E\left(\beta^{\prime}\right)^{2} \rrbracket\langle\psi\rangle\left\langle E \partial_{x} \psi\right\rangle+2 s \lambda^{2} \varphi(a)\left\langle E\left(\beta^{\prime}\right)^{2}\right\rangle \llbracket \psi \rrbracket\left\langle E \partial_{x} \psi\right\rangle+2 s \lambda^{2} \varphi(a)\left\langle E\left(\beta^{\prime}\right)^{2}\right\rangle\langle\psi\rangle \llbracket E \partial_{x} \psi \rrbracket .
\end{aligned}
$$

We use the conditions verified by $\psi$ and $E \partial_{x} \psi$ to obtain the following expression

$$
\begin{aligned}
& 2 s \lambda^{2} \varphi(a) \llbracket E\left(\beta^{\prime}\right)^{2} \psi E \partial_{x} \psi \rrbracket \\
& =\left(\frac{1}{2}-s^{3} \lambda^{4} k \varphi^{3}(a) \mathcal{Q}(a) \llbracket E\left(\beta^{\prime}\right)^{2} \rrbracket\left\langle E \beta^{\prime}\right\rangle \llbracket E \beta^{\prime} \rrbracket+\frac{1}{2} s^{4} \lambda^{5} k^{2} \varphi^{4}(a) \mathcal{Q}^{2}(a) \llbracket E\left(\beta^{\prime}\right)^{2} \rrbracket\left\langle E \beta^{\prime}\right\rangle^{3}\right. \\
& \left.+2 s^{2} \lambda^{3} \varphi^{2}(a)\left\langle E\left(\beta^{\prime}\right)^{2}\right\rangle \llbracket E \beta^{\prime} \rrbracket-2 s^{3} \lambda^{4} k \varphi^{3}(a) \mathcal{Q}(a)\left\langle E\left(\beta^{\prime}\right)^{2}\right\rangle\left\langle E \beta^{\prime}\right\rangle^{2}\right)\langle\psi\rangle^{2} \\
& +\left(\frac{1}{2} s^{2} \lambda^{3} k^{2} \varphi^{2}(a) \mathcal{Q}^{2}(a) \llbracket E\left(\beta^{\prime}\right)^{2} \rrbracket\left\langle E \beta^{\prime}\right\rangle+2 s \lambda^{2} k \varphi(a) \mathcal{Q}(a)\left\langle E\left(\beta^{\prime}\right)^{2}\right\rangle\right)\left\langle E \partial_{x} \psi\right\rangle^{2} \\
& +\left(\frac{1}{2} s^{2} \lambda^{3} k \varphi^{2}(a) \mathcal{Q}(a) \llbracket E\left(\beta^{\prime}\right)^{2} \rrbracket \llbracket E \beta^{\prime} \rrbracket-s^{3} \lambda^{4} k^{2} \varphi^{3}(a) \mathcal{Q}^{2}(a) \llbracket E\left(\beta^{\prime}\right)^{2} \rrbracket\left\langle E \beta^{\prime}\right\rangle^{2}+2 s \lambda^{2} \varphi(a) \llbracket E\left(\beta^{\prime}\right)^{2} \rrbracket\right)\langle\psi\rangle\left\langle E \partial_{x} \psi\right\rangle \\
& +\frac{1}{2} s^{2} \lambda^{3} \varphi^{2}(a) \mathcal{Q}^{2}(a)\left\langle E \beta^{\prime}\right\rangle \llbracket E\left(\beta^{\prime}\right)^{2} \rrbracket\left|g_{s}(a)\right|^{2}-s^{3} \lambda^{4} k \varphi^{3}(a) \mathcal{Q}^{2}(a) \llbracket E\left(\beta^{\prime}\right)^{2} \rrbracket\left\langle E \beta^{\prime}\right\rangle^{2}\langle\psi\rangle g_{s}(a) \\
& +\frac{1}{2} s^{2} \lambda^{3} \varphi^{2}(a) \mathcal{Q}(a) \llbracket E\left(\beta^{\prime}\right)^{2} \rrbracket \llbracket E \beta^{\prime} \rrbracket\langle\psi\rangle g_{s}(a)+2 s^{2} \lambda^{3} \varphi^{2}(a) \mathcal{Q}(a)\left\langle E\left(\beta^{\prime}\right)^{2}\right\rangle\left\langle E \beta^{\prime}\right\rangle\langle\psi\rangle g_{s}(a) \\
& +s^{2} \lambda^{3} k \varphi^{2}(a) \mathcal{Q}^{2}(a)\left\langle E \beta^{\prime}\right\rangle \llbracket E\left(\beta^{\prime}\right)^{2} \rrbracket\left\langle E \partial_{x} \psi\right\rangle g_{s}(a)+2 s \lambda^{2} \varphi(a) \mathcal{Q}(a)\left\langle E\left(\beta^{\prime}\right)^{2}\right\rangle\left\langle E \partial_{x} \psi\right\rangle g_{s}(a) .
\end{aligned}
$$


We estimate the above term with the consideration that $\frac{1}{1+\frac{1}{4} s \lambda k \varphi(a) \llbracket E \beta^{\prime} \rrbracket} \leq \frac{4}{s \lambda k \varphi(a) \llbracket E \beta^{\prime} \rrbracket}$ for $s \lambda$ sufficiently large

$$
\begin{aligned}
& 2 s \lambda^{2} \varphi(a) \llbracket E\left(\beta^{\prime}\right)^{2} \psi E \partial_{x} \psi \rrbracket \\
& \leq\left(2 s^{2} \lambda^{3} \varphi^{2}(a) \llbracket E\left(\beta^{\prime}\right)^{2} \rrbracket\left\langle E \beta^{\prime}\right\rangle+8 s^{2} \lambda^{3} \varphi^{2}(a) \frac{\llbracket E\left(\beta^{\prime}\right)^{3} \rrbracket\left\langle E \beta^{\prime}\right\rangle^{3}}{\llbracket E \beta^{\prime} \rrbracket^{2}}+2 s^{2} \lambda^{3} \varphi^{2}(a)\left\langle E\left(\beta^{\prime}\right)^{2}\right\rangle \llbracket E \beta^{\prime} \rrbracket\right. \\
& \left.+8 s^{2} \lambda^{3} \varphi^{2}(a) \frac{\left\langle E\left(\beta^{\prime}\right)^{2}\right\rangle\left\langle E \beta^{\prime}\right\rangle^{2}}{\llbracket E \beta^{\prime} \rrbracket}\right)\langle\psi\rangle^{2}+\left(8 \lambda \frac{\llbracket E\left(\beta^{\prime}\right)^{2} \rrbracket\left\langle E \beta^{\prime}\right\rangle}{\llbracket E \beta^{\prime} \rrbracket^{2}}+8 \lambda \frac{\left\langle E\left(\beta^{\prime}\right)^{2}\right\rangle}{\llbracket E \beta^{\prime} \rrbracket}\right)\left\langle E \partial_{x} \psi\right\rangle^{2} \\
& +\left(4 s \lambda^{2} \varphi(a) \llbracket E\left(\beta^{\prime}\right)^{2} \rrbracket+16 s \lambda^{2} \varphi(a) \frac{\llbracket E\left(\beta^{\prime}\right)^{2} \rrbracket\left\langle E \beta^{\prime}\right\rangle^{2}}{\llbracket E \beta^{\prime} \rrbracket^{2}}\right)\langle\psi\rangle\left\langle E \partial_{x} \psi\right\rangle \\
& +\frac{8 \lambda\left\langle E \beta^{\prime}\right\rangle \llbracket E\left(\beta^{\prime}\right)^{2} \rrbracket}{k^{2} \llbracket E \beta^{\prime} \rrbracket^{2}}\left|g_{s}(a)\right|^{2}+\frac{s \lambda^{2} \varphi(a)}{k}\left(\frac{16 \llbracket E\left(\beta^{\prime}\right)^{2} \rrbracket\left\langle E \beta^{\prime}\right\rangle^{2}}{\llbracket E \beta^{\prime} \rrbracket^{2}}+2 \llbracket E\left(\beta^{\prime}\right)^{2} \rrbracket+\frac{8\left\langle E\left(\beta^{\prime}\right)^{2}\right\rangle\left\langle E \beta^{\prime}\right\rangle}{\llbracket E \beta^{\prime} \rrbracket}\right)\langle\psi\rangle g_{s}(a) \\
& +\left(\frac{16 \lambda\left\langle E \beta^{\prime}\right\rangle \llbracket E\left(\beta^{\prime}\right)^{2} \rrbracket}{k \llbracket E \beta^{\prime} \rrbracket^{2}}+\frac{8 \lambda\left\langle E\left(\beta^{\prime}\right)^{2}\right\rangle}{k \llbracket E \beta^{\prime} \rrbracket}\right)\left\langle E \partial_{x} \psi\right\rangle g_{s}(a) .
\end{aligned}
$$

Using $\varphi(a) \leq \varphi^{3}(a)$, we obtain

$$
\begin{aligned}
& \left|\mathcal{X}_{12}\right| \leq C_{\epsilon}\left(\alpha, e_{2}, \epsilon\right) s \lambda^{4} \int_{\Omega^{\prime}} \varphi^{3}|\psi|^{2} \mathrm{~d} x+\epsilon s \lambda^{2} \int_{\Omega^{\prime}} \varphi\left|\partial_{x} \psi\right|^{2} \mathrm{~d} x+\left(C s^{2} \lambda^{3}+C_{\epsilon} s \lambda^{3}+\epsilon s \lambda^{3}\right) \varphi^{3}(a)\langle\psi\rangle^{2} \\
& +(C \lambda+C \epsilon s \lambda+\epsilon \lambda) \varphi(a)\left\langle E \partial_{x} \psi\right\rangle^{2}+\left(C \lambda+C_{\epsilon} s \lambda \varphi(a)+C_{\epsilon} \lambda\right) \frac{1}{k^{2}}\left|g_{s}(a)\right|^{2} \\
& +s^{2} \lambda^{3} \varphi^{2}(1)|\psi|^{2}(1)+s^{2} \lambda^{3} \varphi^{2}(0)|\psi|^{2}(0)+\lambda\left|E \partial_{x} \psi\right|^{2}(1)+\lambda\left|E \partial_{x} \psi\right|^{2}(0),
\end{aligned}
$$

where $C$ is a constant depending only on the Young moduli and the weight function $\beta$.

The terms $\mathcal{Y}_{j=1,2}$ are estimated as follows

$$
\left|\mathcal{Y}_{1}\right| \leq C_{\epsilon} \frac{s \lambda \varphi(a)}{k^{2}}\left|g_{s}(a)\right|^{2}+\epsilon s \lambda \varphi(a)\left\langle E \partial_{x} \psi\right\rangle^{2}
$$

and

$$
\left|\mathcal{Y}_{2}\right| \leq \frac{C_{\epsilon} s \lambda \varphi(a)}{k^{2}}\left|g_{s}(a)\right|^{2}+\epsilon s^{3} \lambda^{3} \varphi^{3}(a)\langle\psi\rangle^{2}
$$

Similarly, we have

$$
\left\|f_{s}\right\|_{L^{2}\left(\Omega^{\prime}\right)}^{2} \leq C\left\|e^{s \varphi} f\right\|_{L^{2}\left(\Omega^{\prime}\right)}^{2}+C s^{2} \lambda^{4} \int_{\Omega^{\prime}} \varphi^{3}|\psi|^{2} \mathrm{~d} x .
$$

By gathering all the estimates, we get the following inequality

$$
\begin{aligned}
& \left\|\mathcal{P}_{1} \psi\right\|_{L^{2}\left(\Omega^{\prime}\right)}^{2}+\left\|\mathcal{P}_{2} \psi\right\|_{L^{2}\left(\Omega^{\prime}\right)}^{2}+s \lambda^{2} \int_{\Omega^{\prime}} \varphi\left(\beta^{\prime}\right)^{2}\left|E \partial_{x} \psi\right|^{2} \mathrm{~d} x+s^{3} \lambda^{4} \int_{\Omega^{\prime}} \varphi^{3} E^{2}\left(\beta^{\prime}\right)^{4}|\psi|^{2} \mathrm{~d} x \\
& +\mathcal{C} s \lambda \varphi(a)\left\langle E \partial_{x} \psi\right\rangle^{2}+\mathcal{C} s^{3} \lambda^{3} \varphi^{3}(a)\langle\psi\rangle^{2}+\frac{1}{2} s^{3} \lambda^{3} \varphi^{3}(a) \mathcal{Q}^{2}(a)\left(2 \llbracket \beta^{\prime} \rrbracket\left\langle E \beta^{\prime}\right\rangle^{2}+\frac{1}{4} \llbracket \beta^{\prime} \rrbracket \llbracket E \beta^{\prime} \rrbracket^{2}\right)\left|g_{s}(a)\right|^{2} \\
& \leq C\left\|e^{s \varphi} f\right\|_{L^{2}\left(\Omega^{\prime}\right)}^{2}+\left(s^{2} \lambda^{4}+s^{3} \lambda^{3}+C_{\epsilon} s \lambda^{4}\right) \int_{\Omega^{\prime}} \varphi^{3}|\psi|^{2} \mathrm{~d} x+\left(s \lambda+\epsilon s \lambda^{2}\right) \int_{\Omega^{\prime}} \varphi\left|\partial_{x} \psi\right|^{2} \mathrm{~d} x \\
& \left(C s^{2} \lambda^{3}+C_{\epsilon} s \lambda^{3}+\epsilon s \lambda^{3}+\epsilon s \lambda^{3}+\epsilon s^{3} \lambda^{3}\right) \varphi^{3}(a)\langle\psi\rangle^{2}+(C \lambda+C \epsilon s \lambda+\epsilon \lambda+\epsilon \lambda+\epsilon s \lambda) \varphi(a)\left\langle E \partial_{x} \psi\right\rangle^{2} \\
& +\left(C \lambda+C_{\epsilon} s \lambda+C_{\epsilon} \lambda+C \lambda+C_{\epsilon} s \lambda+C_{\epsilon} \lambda+C_{\epsilon} s \lambda\right) \frac{1}{k^{2}} \varphi(a)\left|g_{s}(a)\right|^{2} \\
& +(s \lambda+\lambda) \varphi(1)\left|E \partial_{x} \psi\right|^{2}(1)+(s \lambda+\lambda) \varphi(0)\left|E \partial_{x} \psi\right|^{2}(0) \\
& +\left(s^{3} \lambda^{3}+s^{2} \lambda^{3}\right) \varphi^{3}(1)|\psi|^{2}(1)+\left(s^{3} \lambda^{3}+s^{2} \lambda^{3}\right) \varphi^{3}(0)|\psi|^{2}(0) .
\end{aligned}
$$


By choosing $\epsilon$ sufficiently small and taking $\lambda \geq \lambda_{0}, s \geq s_{0}$, we obtain

$$
\begin{aligned}
& \left\|\mathcal{P}_{1} \psi\right\|_{L^{2}\left(\Omega^{\prime}\right)}^{2}+\left\|\mathcal{P}_{2} \psi\right\|_{L^{2}\left(\Omega^{\prime}\right)}^{2}+s \lambda^{2} \int_{\Omega^{\prime}} \varphi\left|\partial_{x} \psi\right|^{2} \mathrm{~d} x+s^{3} \lambda^{4} \int_{\Omega^{\prime}} \varphi^{3}|\psi|^{2} \mathrm{~d} x+s^{3} \lambda^{3} \varphi^{3}(a)\langle\psi\rangle^{2} \\
& +s \lambda \varphi(a)\left\langle E \partial_{x} \psi\right\rangle^{2}+\frac{1}{2} s^{3} \lambda^{3} \varphi^{3}(a) \mathcal{Q}^{2}(a)\left(\llbracket \beta^{\prime} \rrbracket\left\langle E \beta^{\prime}\right\rangle^{2}+\llbracket E^{2}\left(\beta^{\prime}\right)^{3} \rrbracket\right)\left|g_{s}(a)\right|^{2} \\
& \leq C\left\|e^{s \varphi} f\right\|_{L^{2}\left(\Omega^{\prime}\right)}^{2}+\frac{s \lambda \varphi(a)}{k^{2}}\left|g_{s}(a)\right|^{2}+s \lambda \varphi(1)\left|E \partial_{x} \psi\right|^{2}(1)+s \lambda \varphi(0)\left|E \partial_{x} \psi\right|^{2}(0) \\
& +s^{3} \lambda^{3} \varphi^{3}(1)|\psi|^{2}(1)+s^{3} \lambda^{3} \varphi^{3}(0)|\psi|^{2}(0) .
\end{aligned}
$$

\subsection{Carleman's inequality}

Recalling that $\psi=e^{s \varphi} q$, we have

$$
e^{s \varphi} \partial_{x} q=\partial_{x} \psi-s \lambda \varphi \beta^{\prime} \psi \quad \text { in } \Omega^{\prime}
$$

which yields

$$
s \lambda^{2} \varphi e^{2 s \varphi}\left|\partial_{x} q\right|^{2} \leq C s \lambda^{2} \varphi\left|\partial_{x} \psi\right|^{2}+C s^{3} \lambda^{4} \varphi^{3}|\psi|^{2} \quad \text { in } \Omega^{\prime},
$$

to be used on the left-hand side of (5). Consequently, we obtain

$$
\begin{aligned}
& \left\|\mathcal{P}_{1} \psi\right\|_{L^{2}\left(\Omega^{\prime}\right)}^{2}+\left\|\mathcal{P}_{2} \psi\right\|_{L^{2}\left(\Omega^{\prime}\right)}^{2}+s \lambda^{2} \int_{\Omega^{\prime}} \varphi e^{2 s \varphi}\left|\partial_{x} q\right|^{2} \mathrm{~d} x+s^{3} \lambda^{4} \int_{\Omega^{\prime}} \varphi^{3} e^{2 s \varphi}|q|^{2} \mathrm{~d} x+s^{3} \lambda^{3} \varphi^{3}(a) e^{2 s \varphi}\langle q\rangle^{2} \\
& +s \lambda \varphi(a) e^{2 s \varphi}\left\langle E \partial_{x} q\right\rangle^{2}+\frac{1}{4} s \lambda \varphi(a) e^{2 s \varphi} \llbracket q \rrbracket^{2}+\frac{1}{2} s^{3} \lambda^{3} \varphi^{3}(a) \mathcal{Q}^{2}(a)\left(\llbracket \beta^{\prime} \rrbracket\left\langle E \beta^{\prime}\right\rangle^{2}+\llbracket E^{2}\left(\beta^{\prime}\right)^{3} \rrbracket\right) e^{2 s \varphi}|g|^{2} \\
& \leq C\left\|e^{s \varphi} f\right\|_{L^{2}\left(\Omega^{\prime}\right)}^{2}+\frac{s \lambda \varphi(a)}{k^{2}} e^{2 s \varphi(a)}|g|^{2}+s \lambda \varphi(1) e^{2 s \varphi(1)}\left|E \partial_{x} q\right|^{2}(1)+s \lambda \varphi(0) e^{2 s \varphi(0)}\left|E \partial_{x} q\right|^{2}(0) \\
& +s^{3} \lambda^{3} \varphi^{3}(1) e^{2 s \varphi(1)}|q|^{2}(1)+s^{3} \lambda^{3} \varphi^{3}(0) e^{2 s \varphi(0)}|q|^{2}(0)
\end{aligned}
$$

Remark 4. The Carleman estimate, established here, gives the unique continuation for the $1 D$ elasticity equation with an imperfect interface from the knowledge of the boundary data on both sides.

\section{Stability of the inverse problem}

Supposing $q_{i}$ as a solution satisfying $(1,2)$ associated to $k=k_{i}$, for $i=1,2$ and also $f(x)=0$ for all $x \in \Omega^{\prime}$. Let $q$ be the difference between both solutions $q_{1}$ and $q_{2}$ which satisfies the following boundary condition at the point $a$

$$
\llbracket E \partial_{x} q \rrbracket=0, \quad \llbracket q \rrbracket=k_{2}\left\langle E \partial_{x} q\right\rangle+\left(k_{1}-k_{2}\right)\left\langle E \partial_{x} q_{1}\right\rangle .
$$

We apply the Carleman inequality, Theorem (1), to the inverse problem presented above with the interface term $g=\left(k_{1}-k_{2}\right)\left\langle E \partial_{x} q_{1}\right\rangle$ and we get

$$
\begin{aligned}
& \left\|\mathcal{P}_{1} \psi\right\|_{L^{2}\left(\Omega^{\prime}\right)}^{2}+s^{3} \lambda^{4} \int_{\Omega^{\prime}} e^{2 s \varphi} \varphi^{2}|q|^{2} \mathrm{~d} x \\
& \leq\left\|\mathcal{P}_{1} \psi\right\|_{L^{2}\left(\Omega^{\prime}\right)}^{2}+\left\|\mathcal{P}_{2} \psi\right\|_{L^{2}\left(\Omega^{\prime}\right)}^{2}+s \lambda^{2} \int_{\Omega^{\prime}} e^{2 s \varphi} \varphi\left|E \partial_{x} q\right|^{2} \mathrm{~d} x+s^{3} \lambda^{4} \int_{\Omega^{\prime}} e^{2 s \varphi} \varphi^{3}|q|^{2} \mathrm{~d} x \\
& \leq \text { obser }+C \frac{s \lambda \varphi(a)}{k_{2}^{2}} e^{2 s \varphi(a)}|g|^{2}(a)
\end{aligned}
$$

where

obser $=s \lambda \varphi(1) e^{2 s \varphi(1)}\left|E \partial_{x} q\right|^{2}(1)+s \lambda \varphi(0) e^{2 s \varphi(0)}\left|E \partial_{x} q\right|^{2}(0)+s^{3} \lambda^{3} \varphi^{3}(1) e^{2 s \varphi(1)}|q|^{2}(1)+s^{3} \lambda^{3} \varphi^{3}(0) e^{2 s \varphi(0)}|q|^{2}(0)$. 
Using the fact that $\psi=e^{s \varphi} q$, one develops the first term of the above inequality as follows

$$
\begin{aligned}
\mathcal{P}_{1} \psi & =-\partial_{x}\left(E \partial_{x} \psi\right)-s^{2} \lambda^{2} \varphi^{2}\left(\beta^{\prime}\right)^{2} E \psi \\
& =-\partial_{x}\left(E \partial_{x}\left(e^{s \varphi} q\right)\right)-s^{2} \lambda^{2} \varphi^{2}\left(\beta^{\prime}\right)^{2} E e^{s \varphi} q \\
& =-\left(s \lambda\left(E \beta^{\prime}\right)^{\prime} \varphi+s \lambda^{2} E\left(\beta^{\prime}\right)^{2} \varphi^{2}+2 s^{2} \lambda^{2} E\left(\beta^{\prime}\right)^{2} \varphi^{2}\right) e^{s \varphi} q-2 s \lambda \beta^{\prime} \varphi e^{s \varphi} E \partial_{x} q,
\end{aligned}
$$

with the fact that $\partial_{x}\left(E \partial_{x} q\right)=0$.

From (7), we get the following estimate

$$
-\left(s \lambda^{2} E\left(\beta^{\prime}\right)^{2} \varphi^{2}+2 s^{2} \lambda^{2} E\left(\beta^{\prime}\right)^{2} \varphi^{2}\right) e^{s \varphi} q-2 s \lambda \beta^{\prime} \varphi e^{s \varphi} E \partial_{x} q=\mathcal{P}_{1} \psi+s \lambda\left(E \beta^{\prime}\right)^{\prime} \varphi e^{s \varphi} q .
$$

Now, we estimate the last equality (8) using Cauchy's inequality as follows

$$
\begin{aligned}
& \left\|\left(s \lambda^{2} E\left(\beta^{\prime}\right)^{2} \varphi^{2}+2 s^{2} \lambda^{2} E\left(\beta^{\prime}\right)^{2} \varphi^{2}\right) e^{s \varphi} q+2 s \lambda \beta^{\prime} \varphi e^{s \varphi} E \partial_{x} q\right\|_{L^{2}\left(\Omega^{\prime}\right)}^{2}+s^{3} \lambda^{4} \int_{\Omega^{\prime}} e^{2 s \varphi} \varphi^{2}|q|^{2} \mathrm{~d} x \\
& \leq\left\|\mathcal{P}_{1} \psi+s \lambda\left(E \beta^{\prime}\right)^{\prime} \varphi e^{s \varphi} q\right\|_{L^{2}\left(\Omega^{\prime}\right)}^{2}+s^{3} \lambda^{4} \int_{\Omega^{\prime}} e^{2 s \varphi} \varphi^{2}|q|^{2} \mathrm{~d} x \\
& \leq\left\|\mathcal{P}_{1} \psi\right\|_{L^{2}\left(\Omega^{\prime}\right)}^{2}+C s^{2} \lambda^{2} \int_{\Omega^{\prime}} e^{2 s \varphi} \varphi^{2}|q|^{2} \mathrm{~d} x+s^{3} \lambda^{4} \int_{\Omega^{\prime}} e^{2 s \varphi} \varphi^{2}|q|^{2} \mathrm{~d} x .
\end{aligned}
$$

Then, we remark that the third term in the last inequality (9) absorbs the second one and we get by Carleman's estimate (6) the following estimate

$$
\begin{aligned}
& \left\|\left(s \lambda^{2} E\left(\beta^{\prime}\right)^{2} \varphi^{2}+2 s^{2} \lambda^{2} E\left(\beta^{\prime}\right)^{2} \varphi^{2}\right) e^{s \varphi} q-2 s \lambda \beta^{\prime} \varphi e^{s \varphi} E \partial_{x} q\right\|_{L^{2}\left(\Omega^{\prime}\right)}^{2}+s^{3} \lambda^{4} \int_{\Omega^{\prime}} e^{2 s \varphi} \varphi^{2}|q|^{2} \mathrm{~d} x \\
& \leq\left\|\mathcal{P}_{1} \psi\right\|_{L^{2}\left(\Omega^{\prime}\right)}^{2}+s^{3} \lambda^{4} \int_{\Omega^{\prime}} e^{2 s \varphi} \varphi^{2}|q|^{2} \mathrm{~d} x \\
& \leq \text { obser }+C \frac{s \lambda \varphi(a)}{k_{2}^{2}} e^{2 s \varphi(a)}|g|^{2}(a) .
\end{aligned}
$$

Next, we need to estimate the boundary integral in terms of the domain integral and for that we present the following theorem.

Theorem 3. (for more details see [10], Theorem 1.5.1.10) Let $\Omega$ be a bounded open subset of $\mathbb{R}^{n}$ with a Lipschitz boundary $\Gamma$. Then, there exists a constant $C$ such that

$$
\int_{\Gamma}|u|^{p} d s \leq C\left(\epsilon^{1-\frac{1}{p}} \int_{\Omega}|\nabla u|^{p} d x+\epsilon^{-\frac{1}{p}} \int_{\Omega}|u|^{p}\right) d x
$$

for all $u \in W_{p}^{1}(\Omega)$ and $\left.\epsilon \in\right] 0,1[$. In addition, $C$ is a constant independent of $u$ and $\epsilon$ (defined in Lemma 1.5.1.9 [10]).

We present the above estimation in the following form

$$
\epsilon^{\frac{1}{p}-1} \int_{\Gamma}|u|^{p} \mathrm{~d} s \leq C\left(\int_{\Omega}|\nabla u|^{p} \mathrm{~d} x+\epsilon^{-1} \int_{\Omega}|u|^{p}\right) \mathrm{d} x
$$

and to apply it in our case, we make the following choices

$$
\begin{gathered}
u=s \lambda \varphi e^{s \varphi} q \\
\nabla u=s \lambda^{2} \beta^{\prime} \varphi e^{s \varphi} q+s^{2} \lambda^{2} \beta^{\prime} \varphi^{2} e^{s \varphi} q+s \lambda \varphi e^{s \varphi} \partial_{x} q \\
\epsilon=\left(s \lambda^{2}\right)^{-1}
\end{gathered}
$$


By Theorem 3, we get then the trace inequality

$$
\begin{aligned}
s^{5 / 2} \lambda^{3} e^{2 s \varphi}\left(\left|q\left(a^{+}\right)\right|^{2}+\left|q\left(a^{-}\right)\right|^{2}\right) & \leq s^{5 / 2} \lambda^{3} e^{2 s \varphi}\left(|q(1)|^{2}+|q(0)|^{2}+\left|q\left(a^{+}\right)\right|^{2}+\left|q\left(a^{-}\right)\right|^{2}\right) \\
& \leq \text { obser }+C \frac{s \lambda \varphi(a)}{k^{2}} e^{2 s \varphi(a)}|g|^{2}(a) .
\end{aligned}
$$

We use the expression $\llbracket q \rrbracket=k_{2}\left\langle E \partial_{x} q\right\rangle+g$ to get the following inequality

$$
\begin{aligned}
|g|^{2} & =\left|\llbracket q \rrbracket-k_{2}\left\langle E \partial_{x} q\right\rangle\right|^{2} \\
& \leq 2\left(|\llbracket q \rrbracket|^{2}+\left|k_{2}\left\langle E \partial_{x} q\right\rangle\right|^{2}\right) \\
& \leq 2\left|q\left(a^{+}\right)-q\left(a^{-}\right)\right|^{2}+2\left|k_{2}\left\langle E \partial_{x} q\right\rangle\right|^{2} \\
& \leq 4\left(\left|q\left(a^{+}\right)\right|^{2}+\left|q\left(a^{-}\right)\right|^{2}\right)+2\left|k_{2}\left\langle E \partial_{x} q\right\rangle\right|^{2} .
\end{aligned}
$$

Then, inserting the above inequality in (11), it yields

$$
\begin{aligned}
s^{5 / 2} \lambda^{3} e^{2 s \varphi(a)}|g|^{2} & \leq s \lambda \varphi(1) e^{2 s \varphi(1)}\left|E \partial_{x} q\right|^{2}(1)+s \lambda \varphi(0) e^{2 s \varphi(0)}\left|E \partial_{x} q\right|^{2}(0)+s^{3} \lambda^{3} \varphi^{3}(1) e^{2 s \varphi(1)}|q|^{2}(1) \\
& +s^{3} \lambda^{3} \varphi^{3}(0) e^{2 s \varphi(0)}|q|^{2}(0)+C \frac{s \lambda \varphi}{k^{2}} e^{2 s \varphi(a)}\left|k_{1}-k_{2}\right|^{2}\left\langle\partial_{x} q_{1}\right\rangle^{2}+s^{5 / 2} \lambda^{3} e^{2 s \varphi(a)}\left|k_{2}\left\langle E \partial_{x} q\right\rangle\right|^{2} .
\end{aligned}
$$

We divide all the terms by the factor $s^{5 / 2} \lambda^{3}$

$$
\begin{aligned}
& e^{2 s \varphi(a)}\left|k_{1}-k_{2}\right|^{2}\left\langle E \partial_{x} q_{1}\right\rangle^{2} \\
& \leq \frac{1}{\sqrt{s}} \varphi(1) e^{2 s \varphi(1)}\left|E \partial_{x} q\right|^{2}(1)+\frac{1}{\sqrt{s}} \varphi(0) e^{2 s \varphi(0)}\left|E \partial_{x} q\right|^{2}(0)+\sqrt{s} \varphi^{3}(1) e^{2 s \varphi(1)}|q|^{2}(1) \\
& +\sqrt{s} \varphi^{3}(0) e^{2 s \varphi(0)}|q|^{2}(0)+C \frac{1}{\sqrt{s}} \frac{\varphi(a)}{k^{2}} e^{2 s \varphi(a)}\left|k_{1}-k_{2}\right|^{2}\left\langle\partial_{x} q_{1}\right\rangle^{2}+e^{2 s \varphi(a)}\left|k_{2}\left\langle E \partial_{x} q\right\rangle\right|^{2} .
\end{aligned}
$$

For $s$ large enough, the left-hand side in (12) can absorb the last term of the right-hand side and the first terms of the right-hand side are sufficiently small. Therefore, since $\varphi$ is a bounded function, we obtain

$$
\left|k_{1}-k_{2}\right|^{2}\left\langle E \partial_{x} q_{1}\right\rangle^{2} \leq \mathcal{C}\left(|q|^{2}(1)+|q|^{2}(0)\right)+e^{2 s \varphi(a)}\left|k_{2}\left\langle E \partial_{x} q\right\rangle\right|^{2} .
$$

For $\left\langle E \partial_{x} q_{1}\right\rangle \geq \mathrm{m}>0$, we have the stability estimate

$$
\left|k_{1}-k_{2}\right|^{2} \leq \mathcal{C}\left(\left|q_{1}-q_{2}\right|^{2}(1)+\left|q_{1}-q_{2}\right|^{2}(0)\right)+e^{2 s \varphi(a)}\left|k_{2}\left\langle E \partial_{x} q\right\rangle\right|^{2} .
$$

Now, we estimate the second term by using a trace inequality, $q$ as a harmonic function and the properties of the function $\varphi$

Here, we choose

$$
\|v\|_{L^{2}(\partial \Omega)}^{2} \leq C^{\prime}\|v\|_{H^{1}(\Omega)}^{2} \leq C^{\prime}\|v\|_{L^{2}(\Omega)}^{2} .
$$

$$
v=\varphi^{1 / 2} e^{s \varphi} \partial_{x} q .
$$

So, one gets

$$
s^{5 / 2} \lambda^{3} \varphi(a) e^{2 s \varphi(a)}\left|k_{2}\left\langle E \partial_{x} q\right\rangle\right|^{2} \leq C^{\prime} s^{5 / 2} \lambda^{3}\left\|\varphi^{1 / 2} e^{s \varphi} \partial_{x} q\right\|_{L^{2}(\Omega)}^{2}
$$

and we estimate the right-hand side of the above inequality by Carleman's inequality (1) as following

$$
\begin{aligned}
& s^{5 / 2} \lambda^{3}\left\|\varphi^{1 / 2} e^{s \varphi} \partial_{x} q\right\|_{L^{2}(\Omega)}^{2} \\
& =s^{3 / 2} \lambda\left\{s \lambda^{2}\left\|\varphi^{1 / 2} e^{s \varphi} \partial_{x} q\right\|_{L^{2}(\Omega)}^{2}\right\} \\
& \leq s^{3 / 2} \lambda\left\{s \lambda \varphi(1) e^{2 s \varphi(1)}\left|E \partial_{x} q\right|^{2}(1)+s \lambda \varphi(0) e^{2 s \varphi(0)}\left|E \partial_{x} q\right|^{2}(0)+s^{3} \lambda^{3} \varphi^{3}(1) e^{2 s \varphi(1)}|q|^{2}(1)\right\} \\
& +s^{3 / 2} \lambda\left\{s^{3} \lambda^{3} \varphi^{3}(0) e^{2 s \varphi(0)}|q|^{2}(0)+C \frac{s \lambda \varphi}{k^{2}} e^{2 s \varphi(a)}\left|k_{1}-k_{2}\right|^{2}\left\langle\partial_{x} q_{1}\right\rangle^{2}\right\} .
\end{aligned}
$$


We divide all the terms by $s^{5 / 2} \lambda^{3}$

$$
\begin{aligned}
& \varphi(a) e^{2 s \varphi(a)}\left|k_{2}\left\langle E \partial_{x} q\right\rangle\right|^{2} \\
& \leq \frac{1}{\lambda} \varphi(1) e^{2 s \varphi(1)}\left|E \partial_{x} q\right|^{2}(1)+\frac{1}{\lambda} \varphi(0) e^{2 s \varphi(0)}\left|E \partial_{x} q\right|^{2}(0)+s^{2} \lambda \varphi^{3}(1) e^{2 s \varphi(1)}|q|^{2}(1) \\
& +s^{2} \lambda \varphi^{3}(0) e^{2 s \varphi(0)}|q|^{2}(0)+C \frac{1}{\lambda} \varphi(a) e^{2 s \varphi(a)}\left|k_{1}-k_{2}\right|^{2}\left\langle\partial_{x} q_{1}\right\rangle^{2} .
\end{aligned}
$$

For $\lambda$ large enough, the left-hand side can absorb the last term of the right-hand side and the first terms of the right-hand side are sufficiently small.

Therefore, since $\varphi$ is a bounded function, we obtain

$$
\left|k_{2}\left\langle E \partial_{x} q\right\rangle\right|^{2} \leq C\left\{|q|^{2}(1)+|q|^{2}(0)\right\} .
$$

Remark 5. The main idea for the above proof is presented by two step, the first one by estimating the interfacial term $\left\langle E \partial_{x} q\right\rangle$ by the parameter $s$ and the second one by estimating the interface additional term $\left\langle E \partial_{x} q_{1}\right\rangle$ by the parameter $\lambda$. It is indispensable to write the Carleman estimate in terms of the two parameters $s$ and $\lambda$.

\section{Conclusion}

By borrowing the approach developed by Benabdallah et al. [5], we prove a Carleman-type estimate that quantifies the unique continuation result for the case of one-dimensional elasticity equation with a spring-type linear law. The proof is based on the construction of suitable weight functions, whose gradient is non-zero, the jump of the derivative is positive across the interface and the average of the derivative vanishes which is a new parameter imposed to enable the control of the interface terms. We have proved a stability estimate for the inverse problem of identifying a stiffness parameter from measurements available on both sides of the external boundary. The extendibility of this stability estimate to the case of the non-stationary problem will be pursued in the future. An extension to higher dimensions will be a continuation of this work such that the challenge will be in the reconstruction of the weight function to get a higher dimensional Carleman-type estimate but the stability proof will have the same lines as the one presented in this work.

\section{Acknowledgements}

The author would like to thank Cédric Bellis, Assia Benabdallah and Yves Dermenjian for numerous discussions on the subject of this work. Financial support is acknowledged to the Tunisian Ministry of Higher Education and Scientific Research and to the Laboratory of Mechanics and Acoustics, CNRS, Marseille.

\section{References}

[1] G. Alessandrini, L. Del Piero and L. Rondi (2003), Stable determination of corrosion by a single electrostatic boundary measurement, Inverse Problems, 19, 973-984

[2] Z. Belhachmi and H. Meftahi (2015), Uniqueness and stable determination in the inverse Robin transmission problem with one electrostatic measurement, Mathematical Methods in the Applied Sciences, 38, 221-240

[3] M. Bellassoued, M. Choulli and A. Jbalia (2013), Stability of the determination of the surface impedance of an obstacle from the scattering amplitude, Mathematical Methods in the Applied Sciences, 36, 2429-2448 
[4] A. Benabdallah, Y. Dermenjian and J. Le Rousseau (2006), Carleman estimates for the onedimensional heat equation with a discontinuous coefficient and applications, Comptes Rendus Mécanique, 334, 582-586

[5] A. Benabdallah, Y. Dermenjian and J. Le Rousseau (2007), Carleman estimates for the onedimensional heat equation with a discontinuous coefficient and applications to controllability and an inverse problem, Journal of Mathematical Analysis and Applications, 336, 865-887

[6] Y. Benvenistea and T. Milohb (2001), Imperfect soft and stiff interfaces in two-dimensional elasticity, Mechanics of Materials, 33, 309-323

[7] S. Chaabane and M. Jaoua (1999), Identification of Robin coefficients by the means of boundary measurements, Inverse Problems, 15, 1425-1438

[8] J. Cheng, M. Choulli and J. Lin (2008), Stable determination of a boundary coefficient in an elliptic equation, Mathematical Models and Methods in Applied Sciences, 18,107-123

[9] A. Doubova, A. Osses and J.P. Puel (2002), Exact controllability to trajectories for semilinear heat equations with discontinuous diffusion coefficients, ESAIM: Control, Optimisation and Calculus of Variations, 8, 621-661

[10] P. Grisvard (1985), Elliptic problems in nonsmooth domains. Monographs and studies in Mathematics, Pitman, Boston

[11] M. Jaoua, S. Nicaise and L. Paquet (2003), Identification of cracks with non linear impedances, ESAIM: Mathematical Modelling and Numerical Analysis, 37, 241-257

[12] J.P. Jones and J.S. Whittier (1967), Waves at a flexibly bonded interface, Journal of Applied Mechanics, 34, 905-909

[13] B. Méjri, Identification of Geometric Flaws in Linear Elasticity, PhD University of Tunis El Manar, 2018

[14] N.M. Newmark, C.P. Siess and I.M. Viest (1951), Tests and analysis of composite beams with incomplete interaction, Proceedings of Society for Experimental Stress Analysis, 9, 75-92

[15] A. Pilarski and J.L. Rose (1988), A transverse-wave ultrasonic oblique incidence technique for interracial weakness detection in adhesive bonds, Journal of Applied Physics, 63, 300-307

[16] K.D. Phung (2003), Remarques sur l'observabilitée pour l'équation de Laplace, ESAIM: Control, Optimisation and Calculus of Variations, 9, 621-635

[17] M.L. Raffa, Micromechanical modeling of imperfect interfaces and applications, PhD Universitị Roma Tor Vergata, Aix-Marseille Université, 2015

[18] E. Sincich (2007), Lipschitz stability for the inverse Robin problem, Inverse Problems, 23

[19] M. Yamamoto (2009), Carleman estimates for parabolic equations and applications, Inverse problems, 25 